\title{
Identification of the Anterior Nucleus of the Ansa Lenticularis in Birds as the Homolog of the Mammalian Subthalamic Nucleus
}

\author{
Yun Jiao, ${ }^{1}$ Loreta Medina, ${ }^{2}$ C. Leo Veenman, ${ }^{3}$ Claudio Toledo, ${ }^{4}$ Luis Puelles, ${ }^{2}$ and Anton Reiner ${ }^{1}$ \\ ${ }^{1}$ Department of Anatomy and Neurobiology, University of Tennessee-Memphis, Memphis, Tennessee 38163, ${ }^{2}$ Department \\ of Morphological Sciences, Facultad de Medicina, Universidad de Murcia, Campus de Espinardo, Murcia 30100, Spain, \\ ${ }^{3}$ Department of Pharmacology, Faculty of Medicine, Technion Institute of Technology, Haifa 31096 Israel, and ${ }^{4}$ Department \\ of Physiology and Biophysics, Institute of Biomedical Sciences, University of Sao Paulo, Sao Paulo-SP, 05508-900 Brazil
}

In mammals, the subthalamic nucleus (STN) is a glutamatergic diencephalic cell group that develops in the caudal hypothalamus and migrates to a position above the cerebral peduncle. By its input from the external pallidal segment and projection to the internal pallidal segment, STN plays a critical role in basal ganglia functions. Although the basal ganglia in birds is well developed, possesses the same major neuron types as in mammals, and plays a role in movement control similar to that in mammals, it has been uncertain whether birds possess an STN. We report here evidence indicating that the so-called anterior nucleus of the ansa lenticularis (ALa) is the avian homolog of mammalian STN. First, the avian ALa too develops within the mammillary hypothalamic area and migrates to a position adjacent to the cerebral peduncle. Second, ALa specifically receives input from dorsal pallidal neurons that receive input from enkephalinergic striatal neurons, as is true of STN. Third, ALa projects back to avian dorsal pallidum, as also the case for STN in mammals. Fourth, the neurons of ALa contain glutamate, and the target neurons of ALa in dorsal pallidum possess AMPA-type glutamate receptor profiles resembling those of mammalian pallidal neurons. Fifth, unilateral lesions of ALa yield behavioral disturbances and movement asymmetries resembling those observed in mammals after STN lesions. These various findings indicate that ALa is the avian STN, and they suggest that the output circuitry of the basal ganglia for motor control is similar in birds and mammals.

Key words: striatum; pallidum; motor functions; segmental development; evolution; glutamate
The subthalamic nucleus (STN) in mammals provides an important linkage between the direct and indirect output pathways of the basal ganglia (Albin et al., 1989b; DeLong, 1990; Gerfen, 1992). The STN creates this linkage by the input it receives from the external segment of globus pallidus (GPe) and by its projection, in turn, to the internal segment of globus pallidus (GPi) and the pars reticulata of substantia nigra (SNr) (Albin et al., 1989b; DeLong, 1990). The striato-GPi pathway to motor-related cortical areas via the ventral anterior and ventral lateral thalamic nuclei (VA/VL) is thought to promote planned movements (Albin et al., 1989b; Gerfen, 1992; Reiner, 1994). Similarly, by its projection to cortex via $\mathrm{VA} / \mathrm{VL}$ and by its input to premotor neurons of deep superior colliculus, the striato-SNr circuit also appears to promote planned movements, specifically of head and eye (Albin et al., 1989b; Gerfen, 1992; Reiner, 1994). In contrast, the STN input to GPi and $\mathrm{SNr}$ is thought to suppress unwanted movements (Albin et al., 1989b).

The neurotransmitters used by the neurons of the direct and indirect pathways are critical to the roles of these two circuits. Striatal, pallidal, and $\mathrm{SNr}$ projection neurons are GABAergic, whereas STN neurons are glutamatergic (Kita and Kitai, 1987; Kitai and Kita, 1987; Smith and Parent, 1988; Albin et al., 1989a,b; Graybiel, 1990; Reiner and Anderson, 1990; Gerfen, 1992). Thus, activation of striato-GPi/SNr neurons facilitates movement by disinhibiting VA/VL or collicular neurons. In contrast, activation of striato-GPe neurons inhibits GPe neurons, which disinhibits the

Received March 9, 2000; revised July 3, 2000; accepted July 5, 2000.

This work was supported by National Institutes of Health Grants NS-19620, NS28721, and EY05298 (to A.R.), Human Frontiers Science Program Grant BIO4CT96-0042 (to L.P.), the Fundação de Amparo à Pesquisa do Estado de São Paulo (to C.T.), and Spanish DGICYT Grant PB96-0715 (to L.M.). We thank Sherry Cuthbertson and Adilson Alves for their excellent technical assistance and are grateful to Dr. Harvey J. Karten for providing us with a digital version of his stereotaxic atlas of pigeon brain, which was used in making Figure 1.

Correspondence should be addressed to Dr. Anton Reiner, Department of Anatomy and Neurobiology, College of Medicine, University of Tennessee-Memphis, 855 Monroe Avenue, Memphis, TN 38163. E-mail: areiner@utmem.edu.

Copyright (C) 2000 Society for Neuroscience $0270-6474 / 00 / 206998-13 \$ 15.00 / 0$ glutamatergic neurons of STN and thereby increases excitatory input from STN to GPi and SNr. The end result of activation of striato-GPe neurons is therefore enhanced inhibition of VA/VL neurons by GPi and $\mathrm{SNr}$, and of deep collicular neurons by $\mathrm{SNr}$. It is presumed that the indirect pathway suppresses movements conflicting with the movements being promoted by the striato-GPi and striato-SNr circuits (Reiner et al., 1988; Albin et al., 1990a,b, 1992).

The connectivity and neurotransmitter organization of the avian basal ganglia and its role in movement control closely resemble those in mammals (Reiner et al., 1984; Medina and Reiner, 1995, 1998a; Medina et al., 1999). As in mammals, the avian striatum contains two major populations of projection neurons: those that co-contain the neuropeptide substance P (SP) and GABA and those that co-contain enkephalin $(\mathrm{ENK})$ and GABA (Graybiel, 1990; Reiner et al., 1998a). In mammals, SP-containing $\left(\mathrm{SP}^{+}\right)$ neurons are the origin of the direct striatal output pathways, whereas $\mathrm{ENK}^{+}$neurons are the source of the indirect striatal output pathway (Albin et al., 1989b; DeLong, 1990). The outputs and pharmacological behavior of $\mathrm{SP}^{+}$striatal neurons in birds appear to resemble those in mammals, lending support to the view that direct striatal output pathways are present in birds (Reiner and Anderson, 1990; Medina et al., 1997; Reiner et al., 1998a). The evidence for the presence of an indirect pathway in birds is less clear-cut, in large part because a homolog of STN has not yet been definitively identified.

Nonetheless, previous studies have provided suggestive evidence for the identity of an avian STN. In birds, the pallidum has among its projection targets a nucleus in the subthalamus called the anterior nucleus of the ansa lenticularis (ALa) (Fig. 1) (Karten and Dubbeldam, 1973; Medina and Reiner, 1997). By its topographic location and its apparent projection back to pallidum and to $\mathrm{SNr}$, ALa is reminiscent of mammalian STN (Brauth et al., 1978; Kita and Kitai, 1987; Kitai and Kita, 1987; Albin et al., 1989b; DeLong, 1990; Hazrati and Parent, 1992; Puelles and Medina,1994; Smith et al., 1994; Shink et al., 1996). We therefore undertook an embryological, hodological, neurochemical, and functional analysis of 


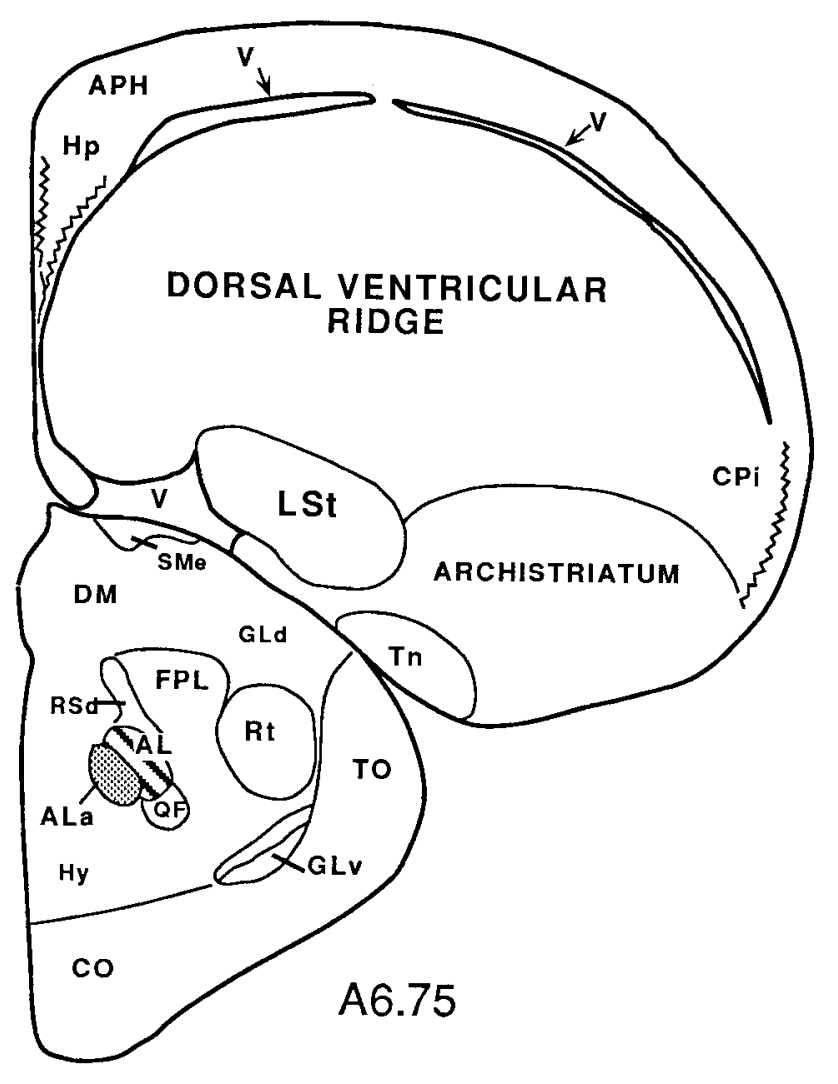

Figure 1. Schematic illustration of a transverse section through the right diencephalon and caudal telencephalon in a pigeon illustrating the location of the anterior nucleus of the ansa lenticularis $(A L a)$ in relation to various other cell groups at that level. The ansa lenticularis $(A L)$ is indicated by diagonal lines, and the ALa is highlighted by stippling. The number at the bottom right represents the anteroposterior level of the section in the stereotaxic coordinates of the Karten and Hodos atlas (1967) for pigeon brain. Medial is to the left, and dorsal to the top. APH, Parahippocampal area; $C O$, optic chiasm; $C P i$, piriform cortex; $D M$, dorsomedial thalamus; $F P L$, lateral forebrain bundle; $G L d$, dorsal lateral geniculate nucleus; $G L v$, ventral lateral geniculate nucleus; $H b$, habenula; $H p$, hippocampus; $H y$, hypothalamus; LSt, lateral striatum; $Q F$, quintofrontal tract; $R S d$, dorsal thalamic reticular nucleus; $R t$, nucleus rotundus; $S M e$, stria medullaris; $T n$, taenia; $T O$, optic tract; $V$, ventricle.

avian ALa to more rigorously assess its possible homology to mammalian STN. Our findings strongly support the view that ALa is homologous to STN.

\section{MATERIALS AND METHODS}

Animal use. Chicks were used in the developmental studies, adult White Carneaux pigeons (Columba livia) were used in the neuroanatomical and behavioral studies, and both adult White Carneaux pigeons and chicks were used in the neurochemical studies. All efforts were made to minimize animal suffering and to reduce the number of animals used, and all animal experiments were done according to institutional (Universities of Tennessee, Murcia, and Sao Paulo) regulations and according to the National Institute of Health Guide for the Care and Use of Laboratory Animals in Research. As an aid, Table 1 presents a list of the terms used for corresponding basal ganglia or basal ganglia-related structures in pigeonschickens and rats.

Developmental studies. Chick embryos from Hamburger and Hamilton (HH) stages $\mathrm{HH} 26-\mathrm{HH} 45$ and hatchlings were used for developmental studies and were staged according to Hamburger and Hamilton (1951). Embryos from stages HH26 to HH35 (i.e., 5-9 d of incubation) were immersed overnight in $4 \%$ paraformaldehyde in $0.1 \mathrm{M}$ sodium phosphate buffer (PB). The heads were then gradually dehydrated and embedded in Paraplast. Chick embryos older than $9 \mathrm{~d}$ of incubation (HH36 and older) and hatchlings were perfused transcardially with $4 \%$ paraformaldehyde in $0.1 \mathrm{M}$ sodium phosphate buffer. Hatchlings were deeply anesthetized using ethyl ether before the transcardial perfusion procedure. After removal, the brains were post-fixed overnight and then gradually dehydrated and embedded in Paraplast. The embryonic heads and brains were sectioned on a microtome at $10-20 \mu \mathrm{m}$ in the sagittal, horizontal, or transverse plane, and sections were mounted, stained with cresyl violet, and coverslipped. At least three series per plane were prepared for each stage and were available for examination in this study.

Neuroanatomical pathway tracing studies. Before surgery, pigeons were deeply anesthetized with ketamine $(66 \mathrm{mg} / \mathrm{kg})$ and xylazine $(33 \mathrm{mg} / \mathrm{kg})$. To investigate the pallidal input to ALa, the dorsal pallidum (also termed paleostriatum primitivum) was successfully injected unilaterally in seven pigeons with $0.15 \mu \mathrm{l}$ of a $5 \%$ solution of biotinylated dextran amine of 10,000 molecular weight (MW) (BDA10k) dissolved in $0.1 \mathrm{M}$ phosphate buffer, $\mathrm{pH} 7.4$, which is transported preferentially in the anterograde direction (Veenman et al., 1992; Medina and Reiner, 1997), using a $1 \mu \mathrm{l}$ Hamilton syringe and procedures described previously. Stereotaxic coordinates for the injections were from the pigeon brain atlas of Karten and Hodos (1967). To investigate the projection of ALa to dorsal pallidum, the dorsal pallidum was successfully injected unilaterally with $0.10 \mu \mathrm{l}$ of a $10 \%$ solution of BDA $3000 \mathrm{MW}$ (BDA3k) in 0.1 M sodium citrate-HCl, pH 3.0, which is transported preferentially in the retrograde direction (Kaneko et al., 1996; Medina et al., 1997), in two pigeons. To investigate the overall efferent projections of ALa, it was successfully injected unilaterally with BDA10k in two pigeons. Note that injections of the anterograde tracer BDA10k in the dorsal pallidum resulted in some retrograde labeling in $\mathrm{ALa}$, whereas injections of the retrograde tracer BDA3k into the dorsal pallidum resulted in some anterograde labeling in ALa. Similarly, injections of the anterograde tracer BDA10k into ALa resulted in some retrograde labeling in the dorsal pallidum. This occurs because, although BDA10k favors anterograde transport and BDA3k favors retrograde transport, neither is a purely unidirectional tracer (Veenman et al., 1992; Fritzsch, 1993; Reiner et al., 1993; Kaneko et al., 1996; Medina and Reiner, 1997).

After $7-10 \mathrm{~d}$, the pigeons were deeply anesthetized with $0.5 \mathrm{ml}$ of $35 \%$ chloral hydrate (intraperitoneally) and perfused transcardially with $30-50$ $\mathrm{ml}$ of $0.1 \mathrm{M}$ sodium phosphate buffer, $\mathrm{pH} 7.4$, containing $6 \%$ dextran, followed by $400 \mathrm{ml}$ of PB containing $4 \%$ paraformaldehyde, $0.01 \mathrm{~m}$ lysine, and $0.1 \mathrm{M}$ sodium periodate (PLP). The brains were then removed and immersed overnight in PB containing $20 \%$ sucrose, $10 \%$ glycerol, and $0.02 \%$ sodium azide. The brains were sectioned frozen on a sliding microtome at $40 \mu \mathrm{m}$ in the frontal plane, and sections were serially collected in PB containing $0.01 \%$ sodium azide as 12 separate evenly spaced series and stored until BDA visualization.

Single-labeling visualization of BDA. Several series of sections (7-10) from the pigeons receiving BDA injections that successfully targeted the dorsal pallidum or ALa were incubated in avidin-biotin complex (ABC) (Vectastain ABC Elite kit; Vector Laboratories, Burlingame, CA), consisting of $5 \mathrm{ml}$ of $\mathrm{PB}$ containing $100 \mu \mathrm{l}$ of avidin $\mathrm{DH}$ and $100 \mu \mathrm{l}$ of biotinylated horseradish peroxidase, for $30 \mathrm{~min}$ at room temperature (Veenman et al., 1992). Sections were then rinsed in PB and immersed in $0.05 \%$ diaminobenzidine tetrahydrochloride (DAB) (Sigma, St. Louis, MO) intensified with $0.04 \%$ nickel ammonium sulfate, $\mathrm{pH} 7.2$, for $10 \mathrm{~min}$. Hydrogen peroxide was then added to the solution to a final concentration of $0.01 \%$, and the sections were reacted in this solution for $10 \mathrm{~min}$. The sections were then mounted on slides, dried, and coverslipped. Several series (four to five series) of the BDA-labeled sections were counterstained with cresyl violet before coverslipping.

Double labeling for BDA and enkephalin or substance $P$. Four to five series of sections from the pigeons receiving injections of BDA10k that successfully targeted ALa were processed by immunofluorescence double labeling to determine whether the retrogradely labeled dorsal pallidal neurons projecting to ALa receive terminals from $\mathrm{ENK}^{+}$or $\mathrm{SP}^{+}$striatal neurons. The BDA-labeled pallidal neurons and either the $\mathrm{ENK}^{+}$or $\mathrm{SP}^{+}$terminals from striatal neurons were simultaneously and differentially labeled using two different fluorophores. The double-labeling procedure has been described in detail previously (Anderson and Reiner, 1990). Tissue immunolabeling was performed by first incubating the tissue for $48-72 \mathrm{hr}$ at $4^{\circ} \mathrm{C}$ in a rabbit polyclonal antiserum against SP or ENK (DiaSorin, Stillwater, $\mathrm{MN}$ ). After rinsing, the tissue was incubated in a cocktail containing a donkey anti-rabbit secondary antiserum conjugated to dichlorotriazinylamino-fluorescein (DTAF) to visualize SP or ENK localization (secondary antisera diluted 1:50; from Jackson ImmunoResearch, West Grove, $\mathrm{PA}$ ) and a mouse monoclonal antibody against biotin conjugated to tetramethylrhodamine isothiocyanate (TRITC) to detect the BDA (1:50 dilution; Jackson ImmunoResearch), for $2 \mathrm{hr}$ at room temperature.

All tissue was then washed three times in PB, mounted on gelatin-coated slides, and coverslipped with $9: 1$ glycerin/0.05 $\mathrm{M}$ carbonate buffer or $9: 1$ glycerol/PBS containing $100 \mathrm{mg} p$-phenylenediamine (Reiner and Anderson, 1990). Sections were examined using an Olympus Optical (Tokyo, Japan) epi-illumination fluorescence microscopy system, using filters that prevent cross-emission of one fluorophore while viewing labeling for the other (Reiner and Anderson, 1990). Sections were also examined using a Bio-Rad (Hercules, CA) MRC-1000 confocal laser scanning microscope (CLSM). For CLSM examination, an Euplan 20 or $40 \times$ Olympus objective was used, and sections were scanned with a krypton-argon laser, with specific excitation wavelength settings selected for TRITC (TRITC setting, $568 \mathrm{~nm}$ ) and DTAF (DTAF setting, $488 \mathrm{~nm}$ ). Sequential collection settings for TRITC emission and DTAF emission were used to capture images for examination and analysis (Medina et al., 1996).

Immunohistochemical single labeling for glutamate or glutamate receptors. Immunohistochemical single labeling was used to determine the distribution of glutamatergic neurons in ALa and characterize the AMPA-type 
Table 1. Basal ganglia terminology in pigeons and rats

\begin{tabular}{|c|c|c|}
\hline Pigeon & Pigeon & Rat \\
\hline (Present paper) & (Karten and Hodos, 1967) & (Paxinos and Watson, 1998) \\
\hline Medial striatum (MSt) & Lobus parolfactorius (LPO) & Striatum or caudoputamen \\
\hline Lateral striatum (LSt) & Paleostriatum augmentatum (PA) & Striatum or caudoputamen \\
\hline Intrapeduncular nucleus (INP) & Intrapeduncular nucleus (INP) & Part of striatum or caudoputamen \\
\hline Dorsal pallidum (DP) & Paleostriatum primitivum (PP) & $\begin{array}{l}\text { Globus pallidus (GP) and entopeduncular } \\
\text { nucleus (EP) }\end{array}$ \\
\hline $\begin{array}{l}\text { Anterior nucleus of the ansa lenticularis } \\
\text { (ALa) }\end{array}$ & $\begin{array}{l}\text { Anterior nucleus of the ansa lenticularis } \\
\text { (ALa) }\end{array}$ & Subthalamic nucleus (STN) \\
\hline Wulst and dorsal ventricular ridge & Wulst and dorsal ventricular ridge & Cerebral cortex \\
\hline $\begin{array}{l}\text { Dorsomedial and dorsointermediate thalamic } \\
\text { nuclei }\end{array}$ & $\begin{array}{l}\text { Dorsomedial and dorsointermediate thalamic } \\
\text { nuclei }\end{array}$ & Intralaminar thalamic nuclei \\
\hline Ventrointermediate thalamic area (VIA) & Not identified as distinct structure & $\begin{array}{l}\text { Ventral anterior and ventral lateral thalamic } \\
\text { nuclei }\end{array}$ \\
\hline
\end{tabular}

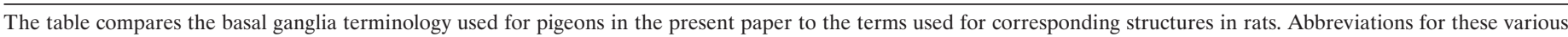

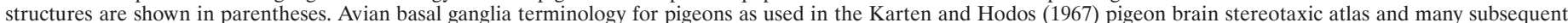

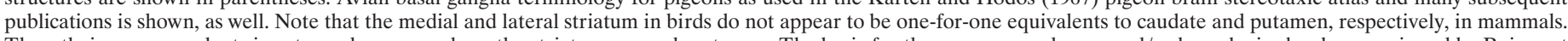

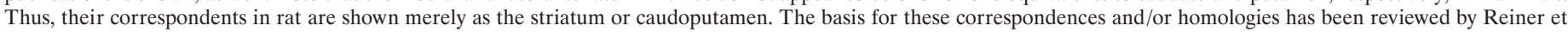
al. (1998a,b). The evidence for the homology of ALa and STN is reviewed in the current paper.

glutamate receptor (GluR) subunits found on pallidal neurons in pigeons and chicks. For these immunohistochemical studies, 12 pigeons were transcardially perfused with the PLP fixative and sectioned at $40 \mu \mathrm{m}$, as described above for the studies using BDA to investigate the connections of ALa. In addition, four adult pigeons and four 2- to 3-week-old chicks were deeply anesthetized and transcardially perfused with $0.9 \%$ saline, followed by $4 \%$ paraformaldehyde in $0.1 \mathrm{M}$ sodium PBS, $\mathrm{pH}$ 7.4. The brains of these birds were removed, post-fixed for $4 \mathrm{hr}$, cryoprotected in $30 \%$ sucrose- $\mathrm{PB}$, sectioned frozen at $35 \mu \mathrm{m}$ using a sliding microtome, and collected as six series.

Sections were incubated in a rabbit anti-glutamate antiserum or in antisera against the GluR1, GluR2/3, or GluR4 AMPA subunits, diluted in PB containing $0.3 \%$ Triton X-100 and $0.01 \%$ sodium azide, for $24-72 \mathrm{hr}$ at $4^{\circ} \mathrm{C}$ under constant gentle agitation. The primary antisera used, their dilutions, and their sources were as follows: (1) a rabbit polyclonal antibody recognizing the GluR1 AMPA receptor subunit, 1:100-1:500 dilution (Chemicon, Temecula, CA); (2) a rabbit polyclonal antibody recognizing an epitope common to the GluR2 and GluR3 AMPA receptor subunits, 1:100-1:250 dilution (Chemicon); (3) a rabbit polyclonal antibody recognizing the GluR4 AMPA receptor subunit, 1:100-1:500 dilution (Chemicon); and (4) a rabbit polyclonal antiserum against glutamate, 1:500-1,000 dilution (generously provided by Aldo Rustioni, University of North Carolina, Chapel Hill, NC or purchased from Arnel Products Co., New York, NY). The antisera against glutamate receptors were generated against synthetic fragments of mammalian glutamate receptor subunits, typically rat. The anti-glutamate receptor antisera and the anti-glutamate antisera have been shown to be specific for their target antigens and to be effective in detecting these antigens (Conti et al., 1987; Chen et al., 1996, 1998). Because GluR1-4 subunits that are highly similar in amino acid sequence to those in mammals have been demonstrated in chicks and pigeons (Ottiger et al., 1995; Paperna et al., 1996; Ravindranathan et al., 1996), it seems highly likely that the anti-GluR used here detected their target antigens in birds.

For the PLP-fixed pigeon tissue, the peroxidase-anti-peroxidase (PAP) method was used to visualize the immunolabeling, whereas for the paraformaldehyde-fixed pigeon and chick tissue, the $\mathrm{ABC}$ method was used. For PAP immunolabeling, after incubation in primary antiserum, sections were rinsed in $\mathrm{PB}$ and incubated for $1 \mathrm{hr}$ in donkey anti-rabbit antiserum (Jackson ImmunoResearch) diluted 1:50, and then in a rabbit peroxidase - anti-peroxidase complex (Sternberger Monoclonals, Baltimore, MD) for $1 \mathrm{hr}$, diluted $1: 100$. The secondary and tertiary antisera were diluted in PB containing $0.3 \%$ Triton X-100 and $0.01 \%$ sodium azide, and the incubations were at room temperature. Sections were then rinsed two times in PB and two times in Tris buffer $(0.05 \mathrm{M}), \mathrm{pH} 7.4$, and immersed in a Tris-buffered solution containing $0.05 \% \mathrm{DAB}$ for $10 \mathrm{~min}$. Hydrogen peroxide was added to the solution to reach a final concentration of $0.01 \%$, and the sections were stained for $10 \mathrm{~min}$, rinsed, mounted, dried, and coverslipped. For the ABC immunolabeling, the ABC Elite kit was used (Vector Laboratories). After incubation in primary antiserum, sections were incubated for $1.5 \mathrm{hr}$ in biotinylated anti-rabbit $\mathrm{IgG}$, washed in $\mathrm{PB}$, and incubated for $1 \mathrm{hr}$ in the $\mathrm{ABC}$ solution. To visualize the labeling, the tissue was preincubated in $0.05 \% \mathrm{DAB}$ for $15 \mathrm{~min}$, followed by additional incubation after addition of $2-5 \mathrm{ml}$ of $0.03 \%$ hydrogen peroxide. These sections were then mounted, lightly osmicated, dried, dehydrated, and coverslipped.

Behavioral studies in pigeons. To analyze the role of ALa in basal ganglia function, ALa, the thalamic region dorsal to it, or nucleus rotundus was targeted for destruction in nine pigeons. The right ALa was targeted in three birds, ALa of both sides was targeted in one bird, the region dorsal to the right ALa was targeted in two birds, and nucleus rotundus was targeted in three birds. These lesions were produced by stereotaxic injection of $2 \mathrm{ng}$ of kainic acid (KA) (Sigma) in $0.5 \mu \mathrm{l}$ of physiological saline, with an infusion speed of $0.02 \mu \mathrm{l} / 5 \mathrm{~min}$ (Piallat et al., 1996). These animals were tested for spontaneous rotation and apomorphine-induced $(6 \mathrm{mg} / \mathrm{kg}$, i.p.) rotation before and after KA injection (Anderson and Reiner, 1990). After completion of the behavioral testing and 3 weeks after the KA lesion, animals were deeply anesthetized by intraperitoneal injection of $35 \%$ chloral hydrate. They were perfused through the left ventricle with $6 \%$ dextran in $0.1 \mathrm{M} \mathrm{PB}, \mathrm{pH} 7.4$, followed by PLP fixative. After $4-6 \mathrm{hr}$ in fixative at $4^{\circ} \mathrm{C}$, brains were stored overnight in $30 \%$ sucrose in phosphate buffer at $\mathrm{pH} 7.4$ and subsequently sectioned frozen at $30-40 \mu \mathrm{m}$ on a sliding microtome. To evaluate the accuracy and the size of the KA lesions, separate series of sections were mounted and stained with cresyl violet or stained immunohistochemically for the glial marker GFAP (DiaSorin), the pan-neuronal marker called the neuron-specific nuclear protein (NeuN) (Chemicon), or glutamate. Antisera dilutions were 1:2000 for the rabbit polyclonal anti-GFAP, 1:500 for the mouse monoclonal anti-NeuN, and 1:500 for the rabbit anti-glutamate. The PAP method using DAB was used to visualize the immunolabeling in these studies and was performed as described above.

\section{RESULTS}

\section{Embryological data}

During development, the neural tube of vertebrates becomes parcellated into transverse, segmental divisions (called neuromeres), as well as into longitudinal divisions (such as the alar and basal plates) (Vaage, 1969; Keyser, 1972; Puelles et al., 1987). The existence of such divisions in the developing brain has received strong support from the expression patterns of numerous homeobox genes that sharply respect the transverse and longitudinal boundaries identified by morphological criteria and appear to contribute to their formation (Bulfone et al., 1993; Puelles and Rubenstein, 1993; Rubenstein et al., 1994, 1998; Shimamura et al., 1995, 1997). The transverse and longitudinal domains appear to be a conserved feature of vertebrate brain organization during development, which implies that putative homologous nervous structures in two different vertebrate groups should develop from corresponding domains (in both the transverse and longitudinal axes) of the brain. In mammals, STN neurons first develop within the mammillary area, which is located within the basal plate of the fourth prosencephalic neuromere (i.e., prosomere 4) (Keyser, 1972; Altman and Bayer, 1978a,b). The STN neurons appear to subsequently migrate dorsally to their adult position close to the alar/ basal boundary near the cerebral peduncle, which itself descends through prosomere 5 (Fig. 2). A standard frontal section of the adult mammalian brain at the level of STN passes obliquely 


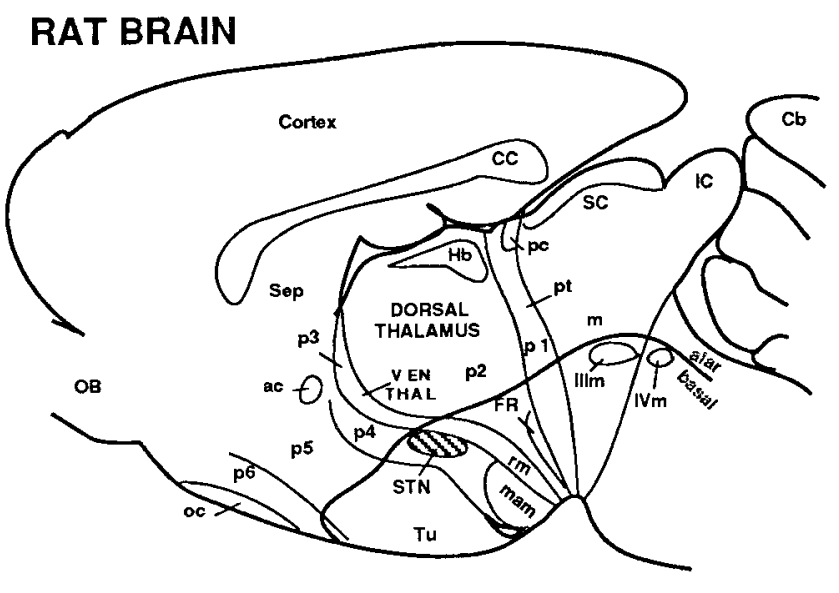

\section{CHICKEN BRAIN}

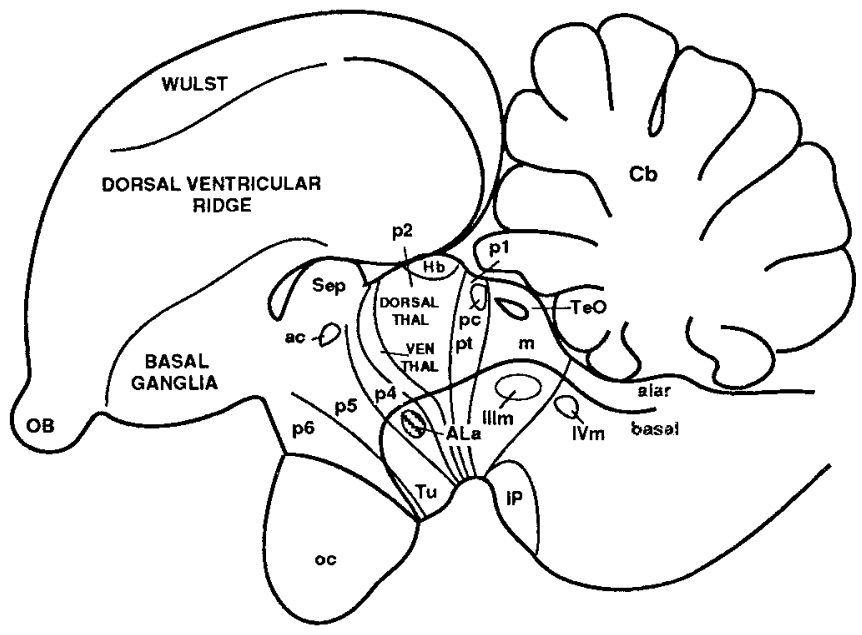

Figure 2. Schematics of sagittal sections through the brains of an adult rat and chicken illustrating the comparable location of the subthalamic nucleus $(S T N)$ of mammals and the anterior nucleus of the ansa lenticularis $(A L a)$ of birds. In these schematics, rostral is to the left, and dorsal is to the top. The location of various forebrain and midbrain cell groups is shown with respect to the segmental boundaries identified in previous studies (see Results). The line of separation between the alar and basal plate is also shown. In this framework, the forebrain is divided into six prosomeres $(p 1-p 6)$ and the midbrain into one mesomere $(m)$, with the first prosomere (p1) abutting the rostral part of the mesomere. ac, Anterior commissure; $C b$, cerebellum; $C C$, corpus callosum; DORSAL THAL, dorsal thalamus; $F R$, fasciculus retroflexus; $H b$, habenula; $I C$, inferior colliculus; $I I I m$, oculomotor nucleus; $I P$, interpeduncular nucleus; $I \mathrm{Vm}$, trochlear nucleus; mam, mammillary region; $o c$, optic chiasm; $O B$, olfactory bulb; $p c$, posterior commissure; $p t$, pretectum; $r m$, retromammillary region; $S C$, superior colliculus; Sep, septum; TeO, optic tectum; $T u$, tuberal hypothalamic region; VEN THAL, ventral thalamus.

through several transverse boundaries, as well as through the alar/ basal longitudinal boundary (Fig. 2), which explains why the STN in such sections seems to be located below the ventral thalamus (which is itself, in fact, located in the alar plate of prosomere 3 ).

In pigeons and chickens, ALa lies medial to the fibers of the ansa lenticularis, which contains the output fibers of the dorsal pallidum (Fig. 1). In conventional transverse sections in the Karten and Hodos (1967) plane, ALa appears to be situated in the rostral diencephalon (Fig. 1). Examination of serial sections through the developing chicken forebrain indicates that the neurons of the avian ALa develop within the basal portion of the fourth prosomere. The position of ALa is also clearly observed in sagittal sections of the chick brain at successive stages, as shown in Figure 3, at stages $\mathrm{HH} 25, \mathrm{HH} 31$, and HH40. The position of ALa with respect to the segmental and longitudinal boundaries of the forebrain during development is schematized in Figure 4. Thus, ALa

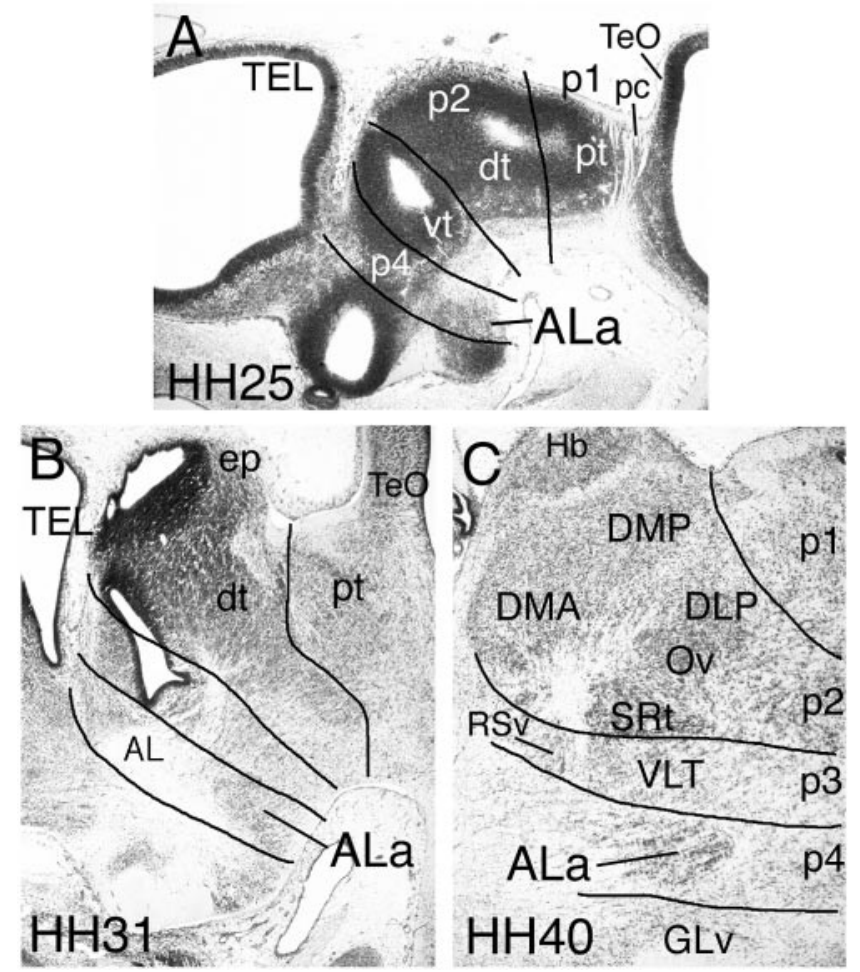

Figure 3. Images of sagittal sections at the level of the mammillary region of embryonic chick brains at developmental stages $\mathrm{HH} 25, \mathrm{HH} 31$, and $\mathrm{HH} 40$. The sections were cresyl violet-stained. In these sections, rostral is to the left, and dorsal is to the top. Note that, at $\mathrm{HH} 25$, the anterior nucleus of the ansa lenticularis $(A L a)$ is located in the basal plate adjacent to the mammillary region within prosomere $4(p 4)$, and at later stages the ALa appears progressively to come to be located more dorsally within prosomere 4. $A L$, Ansa lenticularis; $A L a$, anterior nucleus of the ansa lenticularis; $D L P$, nucleus dorsolateralis posterior thalami; $D M A$, nucleus dorsomedialis anterior thalami; $D M P$, nucleus dorsomedialis posterior thalami; $d t$, dorsal thalamus; $e p$, epithalamus; $G L v$, ventral lateral geniculate nucleus; $H b$, habenula; $O v$, nucleus ovoidalis; $p c$, posterior commissure; $p t$, pretectum; $p 1-p 4$, prosomeres 1 to $4 ; R S v$, nucleus reticularis superior thalami, pars ventralis; $S R t$, nucleus subrotundus; TEL, telencephalon; $T e O$, optic tectum; $v t$, ventral thalamus; $V L T$, nucleus ventrolateralis thalami.

originates in the hypothalamic basal plate of prosomere $4(p 4)$, close to the prospective mammillary body, and gradually migrates dorsally to a position near the alar plate in the same segment. The developmental history and final position of ALa therefore resemble that of the mammalian STN. The identical segmental location of STN and ALa is depicted in schematic drawings comparing adult rat and chicken brains in sagittal view in Figure 2.

\section{Connectivity: pallidal input to ALa}

In birds, the dorsal pallidum appears to contain an intermingling of neurons comparable with those found in GPe and GPi in mammals (Reiner et al., 1998a). Injections of BDA10k centered in the dorsal pallidum of pigeons, which would therefore involve injection into both GPe- and GPi-type neurons, produced abundant anterograde labeling of axonal arborizations and terminals in ALa (Fig. 5A,B). This finding is consistent with previous reports by our group and by others (Karten and Dubbeldam; 1973; Brauth et al., 1978; Medina and Reiner, 1997). Anterograde labeling was also observed in the various other pallidal target areas that have been described previously (Karten and Dubbeldam, 1973; Medina and Reiner, 1997). The morphological traits of the dorsal pallidal neurons projecting to ALa were revealed by injections of BDA10k centered in ALa (see Fig. 9A), which yielded numerous retrogradely labeled neurons in the dorsal pallidum (Fig. $6 A$ ). The BDA-labeled pallidal neurons projecting to ALa possessed large perikarya and smooth dendrites that extended in the medial to lateral axis of the dorsal pallidum. As these dendrites tapered away from their perikarya of origin, they became increasingly varicose $(B)$. 

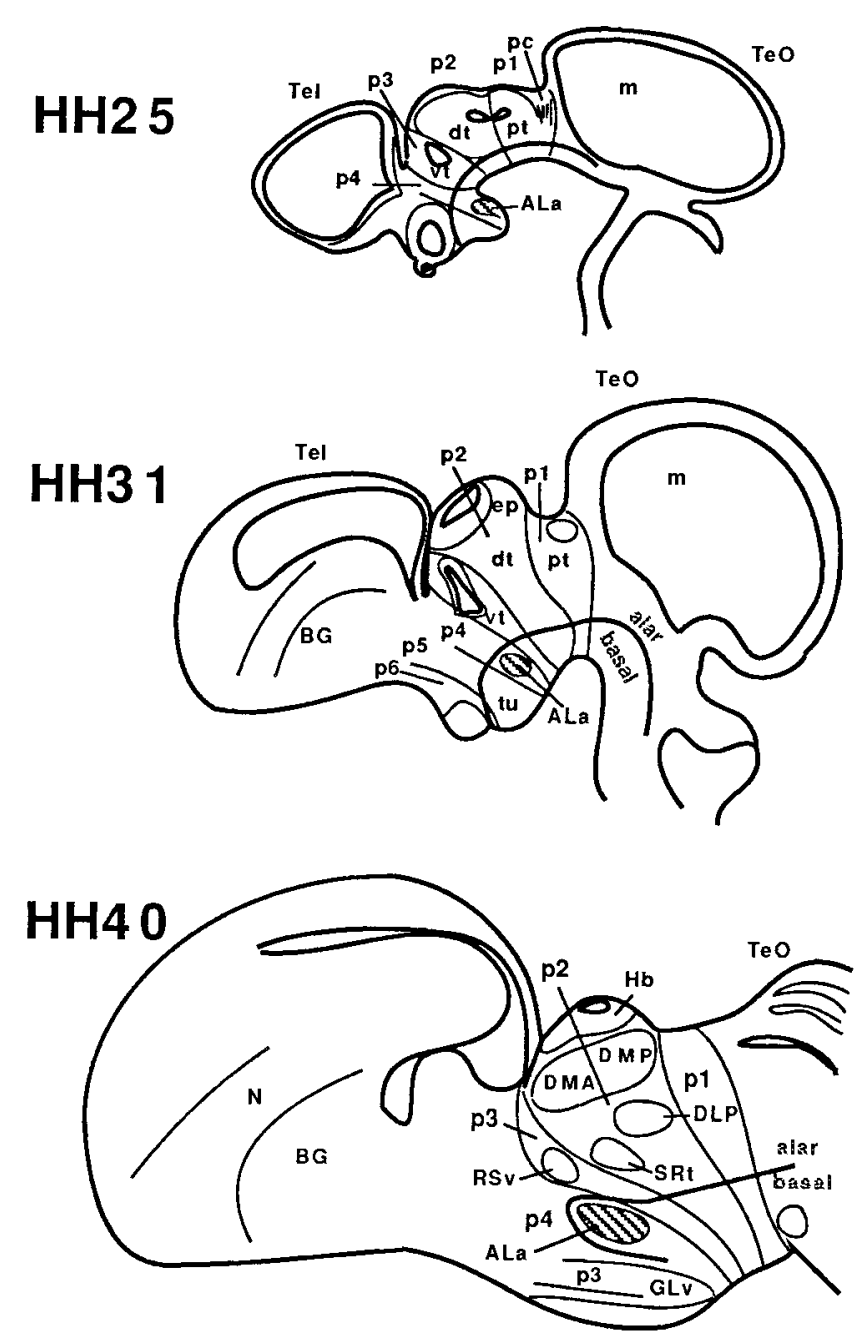

Figure 4. This series of line drawings schematizes the position of ALa in chick embryo at the three progressive stages of development (HH25, HH31, and HH40) shown in Figure 3. Note that ALa originates in the basal plate and migrates to a position near the alar plate within prosencephalic segment $4(p 4)$. ALa, Anterior nucleus of the ansa lenticularis; $B G$, basal ganglia; $D L P$, nucleus dorsolateralis posterior thalami; $D M A$, nucleus dorsomedialis anterior thalami; $D M P$, nucleus dorsomedialis posterior thalami; $d t$, dorsal thalamus; $e p$, epithalamus; $G L v$, ventral lateral geniculate nucleus; $H b$, habenula; $m$, mesomere; $N$, neostriatal subdivision of the dorsal ventricular ridge; $p c$, posterior commissure; $p t$, pretectum; $p 1-p 6$, prosomeres 1 to $6 ; R S v$, nucleus reticularis superior thalami, pars ventralis; $S R t$, nucleus subrotundus; $T e l$, telencephalon; $T e O$, optic tectum; $v t$, ventral thalamus; $t u$, tuberal hypothalamic region.

We performed double-label immunofluorescence combining BDA visualization with ENK or SP immunolabeling to determine whether the ALa-projecting pallidal neurons receive input from $\mathrm{ENK}^{+}$striatal neurons (i.e., are GPe-like) or $\mathrm{SP}^{+}$striatal neurons (i.e., are GPi-like). To provide precise visualization of the terminals and dendrites, we analyzed the results using CLSM. Our results with the double-label immunofluorescence for BDA and ENK showed that all dorsal pallidal neurons projecting to ALa that were examined received $\mathrm{ENK}^{+}$terminals on their cell bodies and dendrites (Fig. 7A-D). In contrast, our results with the double-label immunofluorescence for BDA and SP indicated that none of the BDA-labeled dorsal pallidal neurons projecting to ALa that were examined received $\mathrm{SP}^{+}$terminals on their cell bodies or dendrites (Fig. $7 E, F)$. Thus, the pallidal input to ALa arises specifically from neurons that receive input from $\mathrm{ENK}^{+}$striatal neurons. This is also the case for the STN of mammals, because its pallidal input arises from the GPe, whose neurons primarily receive their striatal input from $\mathrm{ENK}^{+}$neurons (Reiner et al., 1998a, 1999).

\section{Connectivity: ALa projections}

We also confirmed by retrograde and anterograde labeling using $\mathrm{BDA}$ that ALa in pigeons projects back to the dorsal pallidum. After BDA3k injections into the dorsal pallidum, numerous retrogradely labeled neurons were found within ALa (Figs. 5D, 8). The location of the labeled neurons in ALa appeared to vary according to the part of the dorsal pallidum injected with tracer, which suggests that the ALa-pallidal projection is topographically organized. We did not, however, attempt to systematically characterize this topography. Confirming that ALa projects to the dorsal pallidum, BDA10k injection into ALa also yielded extensive anterograde labeling in the dorsal pallidum (Fig. 9B-D). The anterogradely labeled BDA-containing terminals of ALa origin in the dorsal pallidum were clearly observed to contact the Nissl-stained perikarya of pallidal neurons in cresyl violet-counterstained material, but they were not observed to contact the perikarya or dendrites of BDA-labeled pallido-ALa projection neurons (Fig. 9B). In addition, ALa was found to project to two other cell groups in avian brain that are involved in the output circuitry of the avian basal ganglia, namely the lateral spiriform nucleus $(\mathrm{SpL})$ of the pretectum and the SNr of the midbrain tegmentum (Fig. 10). Both the SpL and the SNr appear to be functionally analogous to GPi in that they contain GABAergic neurons, receive direct input from $\mathrm{SP}^{+}$striatal neurons, and have output to premotor regions (Veenman and Reiner, 1994; Medina and Reiner, 1996; Reiner et al., 1998a; Medina et al., 1999).

\section{Glutamate and glutamate receptor localization}

Our immunohistochemical results in pigeons indicate that the neurons of ALa contain the excitatory neurotransmitter glutamate (Fig. 5C). Note that the glutamatergic neurons of ALa lie medial to the ansa lenticularis itself. Glutamatergic neurons lie within the entire rostrocaudal extent of pigeon ALa, and the field of neurons has an $\sim 1 \mathrm{~mm}$ rostrocaudal extent. The distribution of these neurons overlaps the distribution of retrogradely labeled neurons observed in ALa after BDA injections in the dorsal pallidum. Consistent with the glutamatergic nature of the ALa neurons and therefore of the ALa inputs to the dorsal pallidum, SpL, and SNr, many or all of the large neurons in these regions were found to be specifically rich in the GluR1, GluR2/3, and/or GluR4 AMPA-type glutamate receptor subunits in both pigeons and chicks (Fig. 11). Within the dorsal pallidum of pigeon and chick, the vast majority of the medium- to large-sized projection neurons were intensely labeled for GluR4, and many were also moderately or intensely labeled for GluR2/3, but only scattered large pallidal neurons were labeled for GluR1. Within the SNr of pigeon and chick, all or nearly all neurons were rich in GluR4, and many were lightly to moderately labeled for Glu2/3. GluR1 labeling of $\mathrm{SNr}$ neurons was prominent in pigeon but light in chick. Finally, all or nearly all SpL neurons were rich in GluR4 in pigeon and chick and poor in GluR2/3 labeling. GluR1 labeling was moderate in many SpL neurons in pigeon but was primarily absent from $\mathrm{SpL}$ neurons in chick.

\section{Behavioral studies of the effects of ALa lesions}

We found that unilateral lesions that damaged $>25 \%$ of ALa (as confirmed by the postmortem histological analysis) transiently produced hyperkinesia of the opposite wing, spontaneous rotation to the side opposite the lesion, vacuous chewing movements, and jaw tremors. One bird with complete destruction of the left ALa and $62 \%$ destruction of the right ALa showed transient hyperactivity of both wings after the lesions. These behaviors subsided by $4-6 \mathrm{hr}$ after the KA lesion. The effects of unilateral ALa destruction resemble those of unilateral STN damage in rats (Kafetzopoulos and Papadopoulos, 1983; Piallat et al., 1996) in which transient stereotyped extension-flexion movements of the contralateral limbs, orofacial dyskinesias, and spontaneous contraversive rotation were observed after unilateral STN lesions.

During the first week after the KA lesion, the birds were challenged by intraperitoneal injection of apomorphine. Apomorphine 

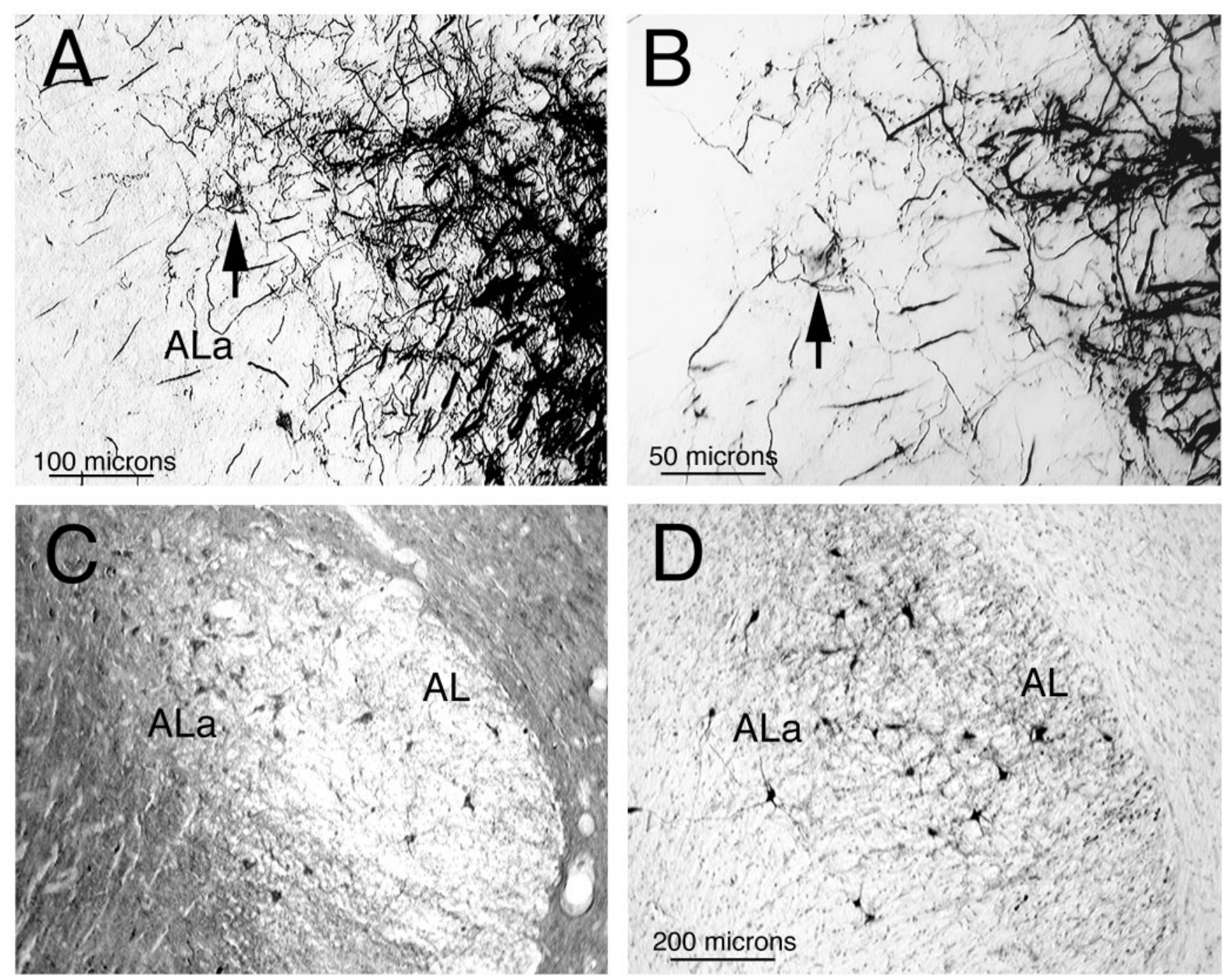

Figure 5. Images from transverse sections showing anterogradely labeled fibers in the anterior nucleus of the ansa lenticularis $(A L a)$ and a few neurons showing light retrograde labeling after a BDA10k injection into the dorsal pallidum, at a low and higher magnification $(A, B)$. The arrows in $A$ and $B$ indicate the same retrogradely labeled neuron. Also shown are images from transverse sections showing neuronal perikarya in ALa immunolabeled for glutamate $(C)$ and retrogradely labeled by BDA3k injection into the dorsal pallidum $(D)$. Note that ALa overlaps the medial edge of the ansa lenticularis $(A L)$. Medial is to the left, and dorsal to the top in all images. $C$ and $D$ are at the same magnification.

is a nonselective dopamine receptor agonist that produces hyperactivity and stereotyped movements. In rats or pigeons with unilateral damage to part of the basal ganglia circuitry, apomorphine produces an asymmetric motor output that manifests itself as rotation. For example, in rats with unilateral destruction of nigral dopaminergic neurons, apomorphine produces rotation to the side opposite the nigral destruction (Hifti et al., 1980; Zeng et al., 1995). This direction of rotation appears to stem, in large part, from the greater apomorphine-mediated activation of the direct $\mathrm{SP}^{+}$ striato-SNr pathway on the dopamine-depleted side because of denervation-produced supersensitivity to apomorphine (HerreraMarschitz and Ungerstedt, 1984; Albin et al., 1989b; Xu et al., 1994; Balk et al., 1995; Hutchison et al., 1997). In contrast, apomorphinetreated rats with STN destruction rotate to the side of the lesion (Kafetzopoulos and Papadopoulos, 1983; Piallat et al., 1996).

The two birds with large unilateral ALa lesions and one bird with complete ALa destruction on one side and partial on the other were found to rotate to the side of the extensive ALa destruction with a frequency of six to seven rotations per minute after apomorphine treatment. The birds with extensive ALa damage also had varying amounts of destruction of either the dorsomedial thalamus, which appears comparable with the intralaminar thalamus of mammals (Veenman et al., 1995, 1997), and/or to nucleus rotundus, which is involved in visual information processing (Ho- dos and Karten, 1966; Karten and Revzin, 1966, 1970). Because of the possibility that damage to these structures could cause or contribute to the rotational behavior, we performed control lesions confined to these structures in several birds. Birds with dorsomedial thalamic lesions that did not damage ALa (two birds) and birds with nucleus rotundus lesions that did not damage ALa (three birds) showed none of the transient dyskinesias characterizing the ALa-lesioned birds, and they only rotated to the lesioned side with a frequency of zero to two rotations per min after apomorphine treatment, which is indistinguishable from the prelesion frequency. The latter results and these differences in apomorphine-induced rotational behavior among the three groups persisted even when the pigeons were blindfolded, which appeared to rule out the possibility that the effects were driven by an asymmetry in visual stimulation. Counts of intact neurons in ALa (from the NeuNimmunolabeled material) allowed us to assess the extent of ALa, dorsomedial thalamus, and nucleus rotundus destruction in each case. We found that the extent of ALa destruction was highly and significantly correlated with the frequency of rotation occurring after the apomorphine injection $(r=0.95)$. In contrast, neither the extent of dorsal thalamic damage nor the extent of nucleus rotundus damage correlated significantly with the apomorphine-induced rotation frequency. 

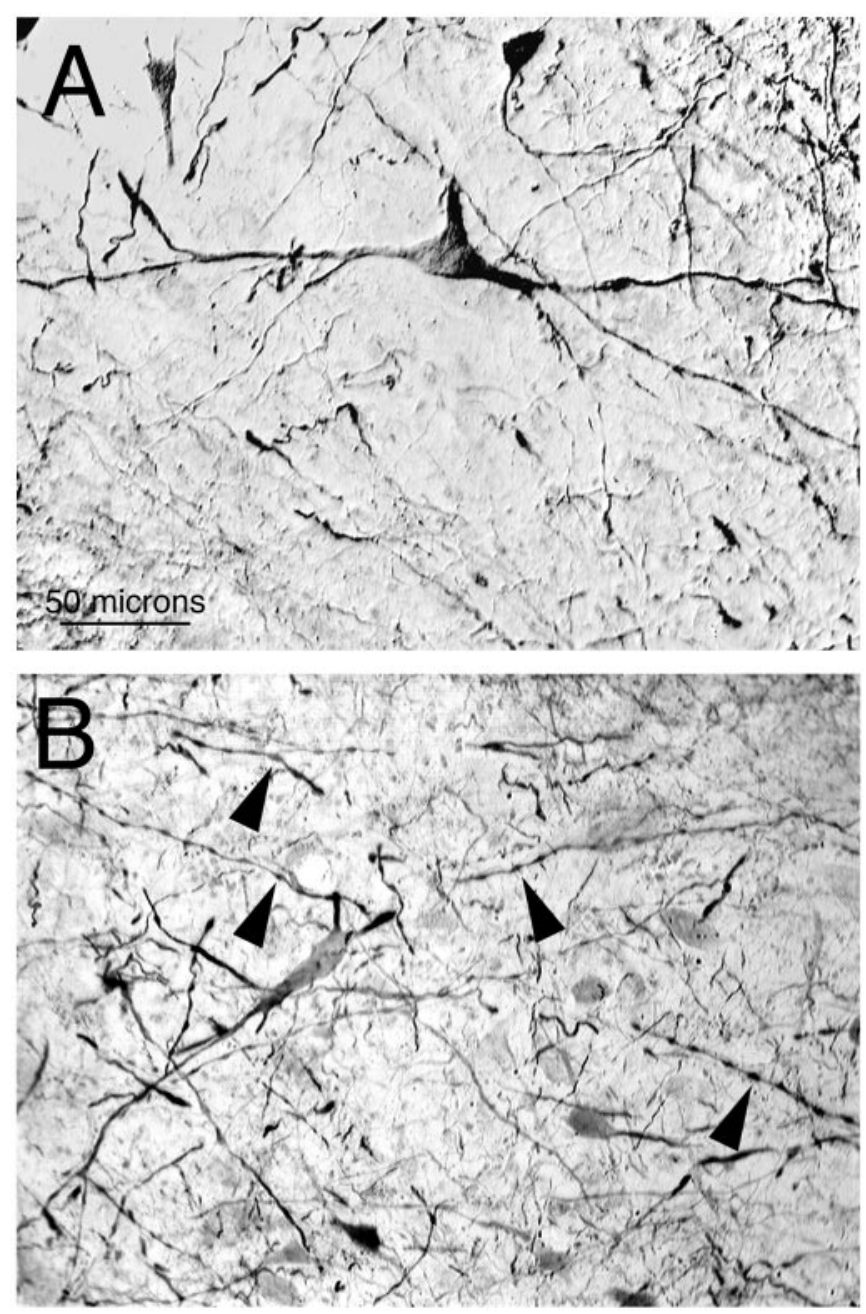

Figure 6. Images from transverse sections showing retrogradely labeled neuronal perikarya, dendrites, and axon terminals in the dorsal pallidum after an injection of BDA10k into ALa. Note that pallido-ALa projection neurons have large perikarya and long smooth dendrites $(A)$ that were observed to become varicose (arrowheads) as they tapered to their tips $(B)$.

\section{DISCUSSION}

The present study provides evidence that the so-called ALa of birds is the homolog of mammalian STN. Our results also indicate that ALa is part of an indirect motor output circuit of the avian basal ganglia, which as in mammals appears to be involved in suppressing unwanted movements. The evidence supporting these conclusions is discussed in the following paragraphs.

\section{ALa and STN develop from an identical brain domain}

Embryological and molecular evidence indicates that the brain in all vertebrate groups consists of an identical number of segmental and longitudinal units, which subdivide the brain into comparable domains (Vaage, 1969; Keyser, 1972; Puelles et al., 1987, 1996, 1999, 2000; Lumsden and Keynes, 1989; Bulfone et al., 1993; Puelles and Rubenstein, 1993; Marin and Puelles, 1994; Rubenstein et al., 1994; Puelles, 1995; Shimamura et al., 1995, 1997; Pombal and Puelles, 1999; Redies et al., 2000). The present study shows that avian ALa and mammalian STN have the same segmental (i.e., prosomere 4) and longitudinal (i.e., basal plate) identity (Fig. 2). This similarity is reinforced by the similarities in neurochemistry, connections, and function also found in the present study. This strongly supports the notion that ALa and STN are homologous.

\section{Circuitry of ALa and STN}

Our previous studies showed that birds possess homologs of the $\mathrm{SP}^{+}$striato-GPi and $\mathrm{SP}^{+}$striato-SNr basal ganglia output channels
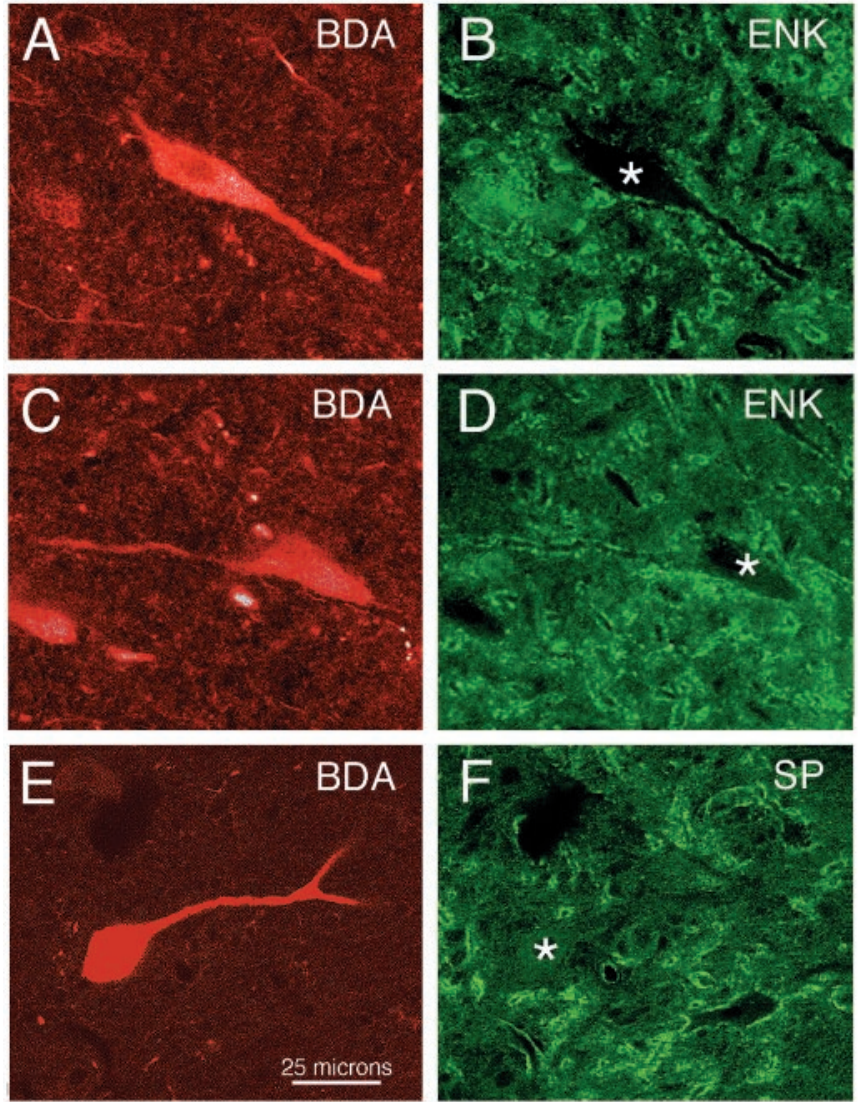

Figure 7. Pallidal neurons projecting to ALa were retrogradely labeled with BDA and visualized using a TRITC-conjugated anti-biotin. In some sections containing TRITC-labeled pallido-ALa neurons, ENK + terminals were labeled with DTAF ( $A$ and $B, C$ and $D)$, whereas in other sections containing TRITC-labeled pallido-ALa neurons, $\mathrm{SP}^{+}$terminals were labeled with DTAF ( $E$ and $F$ ). The images of labeling for ENK or SP with respect to the TRITC-labeled pallido-ALa perikarya shown here were captured using confocal laser scanning microscopy. The two pairs of images showing BDA-ENK double labeling $(A$ and $B, C$ and $D)$ show two separate BDA-labeled pallido-ALa neurons. Note that both of these are surrounded and contacted by $\mathrm{ENK}^{+}$terminals. In contrast, the BDA-labeled pallidoALa neuron in the SP-labeled tissue was not observed to receive $\mathrm{SP}^{+}$ terminals $(E, F)$. Note that none of the $\mathrm{SP}^{+}$striatal terminals in the field contact the BDA-labeled pallidal neuron. The location of the $\mathrm{BDA}^{+}$ perikarya in $A, C$, and $E$ is shown by asterisks in $B, D$, and $F$. The magnification is the same in all images.

found in mammals (Fig. 12) (Medina et al., 1997; Reiner et al., 1998a; Medina and Reiner, 2000). The avian homolog of the former is a circuit that appears to arise from $\mathrm{SP}^{+}$striatal neurons that, in turn, project to dorsal pallidal neurons that project to a ventral tier thalamic region called the ventrointermediate area (VIA) (Medina and Reiner, 1996, 1997; Medina et al., 1997, 1999). The VIA appears to be the avian homolog of mammalian VA/VL based on its position, pallidal input, $\mathrm{SNr}$ input, cerebellar input, and output to a telencephalic motor "cortical" area that appears homologous to mammalian motor cortex (Medina et al., 1997, 1999; Medina and Reiner, 2000). The avian homolog of the mammalian $\mathrm{SP}^{+}$striato-SNr-tectal circuit also arises from $\mathrm{SP}^{+}$striatal neurons, which project to the avian homolog of mammalian $\mathrm{SNr}$, which in turn projects to tectal layers that project to premotor and motor brainstem areas (Reiner and Karten, 1982; Anderson and Reiner, 1991; Anderson et al., 1991; Veenman and Reiner, 1994; Reiner et al., 1998a).

The present findings indicate that birds also possess a basal ganglia output circuit resembling the indirect pathway in mammals, with ALa serving as the STN-like cell group linking the direct and indirect pathways (Fig. 12). We found that ALa receives input from dorsal pallidal neurons that themselves receive input from $\mathrm{ENK}^{+}$ striatal neurons. Both the pallidal neurons projecting to ALa and 

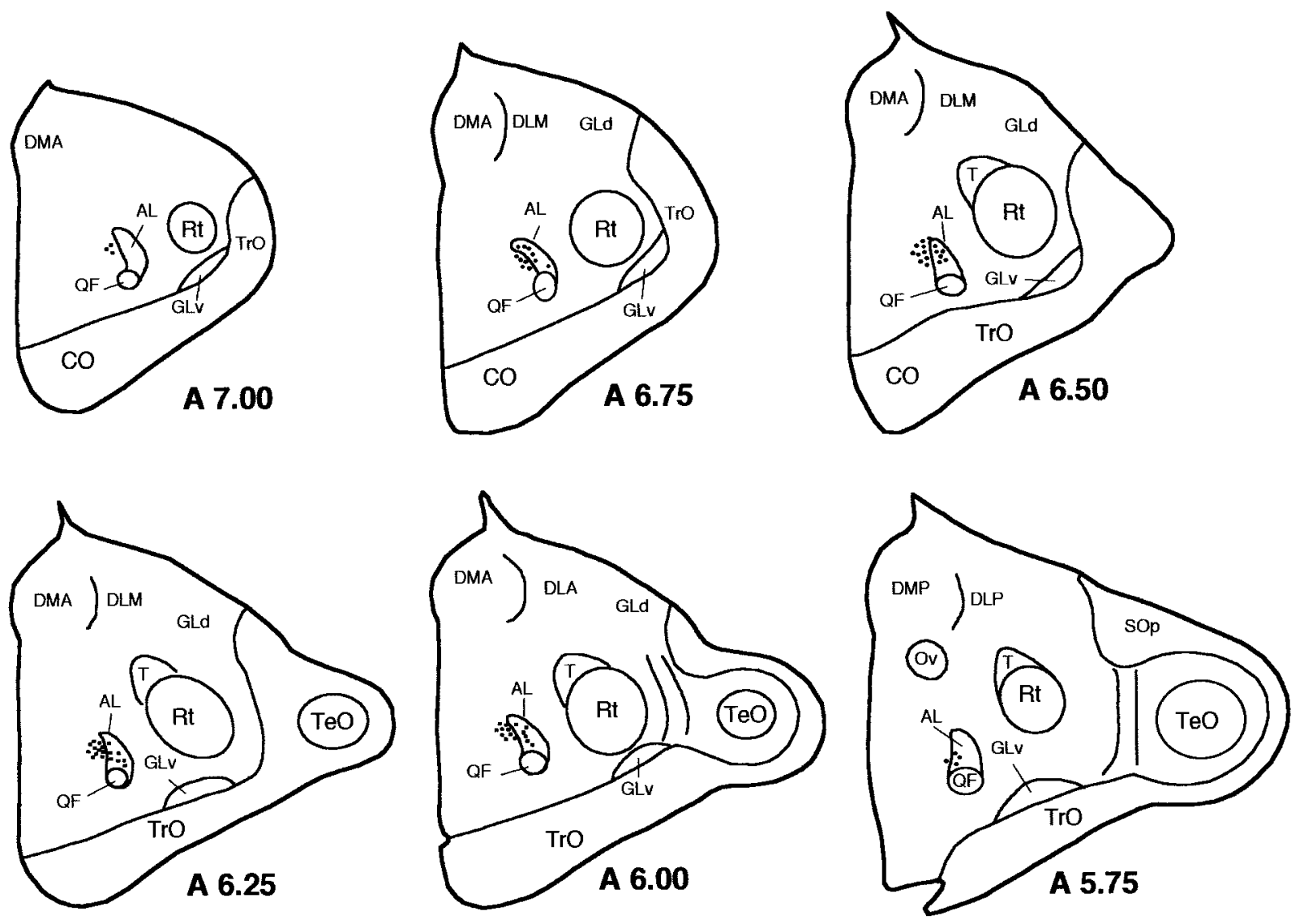

Figure 8. A series of line drawings showing the distribution of retrogradely labeled neurons in pigeon ALa after an injection of BDA3k into the dorsal pallidum (also termed the paleostriatum primitivum). The labeled neurons in ALa are shown as filled circles, and the numbers at the bottom right of each section indicate the anteroposterior level of the sections in the stereotaxic pigeon brain atlas of Karten and Hodos (1967). $A L$, Ansa lenticularis; $C O$, optic chiasm; $D L A$, nucleus dorsolateralis anterior thalami; $D L M$, nucleus dorsolateralis anterior thalami pars medialis; $D L P$, nucleus dorsolateralis posterior thalami; $D M A$, nucleus dorsomedialis anterior thalami; $D M P$, nucleus dorsomedialis posterior thalami; $G L d$, dorsal lateral geniculate nucleus; $G L v$, nucleus geniculatus lateralis pars ventralis; $O v$, nucleus ovoidalis; $Q F$, quintofrontal tract; $R t$, nucleus rotundus; $S O p$, stratum opticum of tectum; $T$, nucleus triangularis; $\mathrm{TeO}$, optic tectum; $\operatorname{Tr} O$, optic tract.

the $\mathrm{ENK}^{+}$neurons projecting to the pallido-ALa neurons appear to be GABAergic, as do the vast majority if not all pallidal and striatal projection neurons in birds (Reiner and Anderson, 1993; Veenman and Reiner, 1994). This is also the case for the homologous neurons in mammals, because the pallidal input to STN arises from GABAergic neurons of $\mathrm{GPe}$, which receive their striatal input predominantly from $\mathrm{ENK}^{+}$GABAergic neurons (Albin et al., 1989b; Reiner and Anderson, 1990). The present study also indicates that ALa neurons are glutamatergic and project back to neurons of the avian dorsal pallidum, which are themselves enriched in AMPA receptor subunits, notably GluR4. In these respects, the ALa-pallidal projection resembles the projection from STN to GPi in mammals (Smith and Parent, 1988; Albin et al., 1989a,b; Petralia and Wenthold, 1992; Bernard et al., 1996; Paquet and Smith, 1996). The avian dorsal pallidum, however, is not organized into segments, and pallidal neurons receiving either $\mathrm{SP}^{+}$ (i.e., GPi-type) or $\mathrm{ENK}^{+}$(i.e., GPe-type) striatal input are intermixed in a single dorsal pallidal field (Medina and Reiner, 1995; Medina et al., 1997, 1999; Reiner et al., 1998a). Although we have not yet directly confirmed that ALa preferentially targets SPrecipient dorsal pallidal neurons that project to VIA, it seems likely this is the case because the fine fibers of the ALa projection to dorsal pallidum were not observed to end on the perikarya or dendrites of ENK-recipient pallido-ALa projection neurons but were observed to end on the perikarya of pallidal neurons that did not project to ALa.

As is true of STN, ALa additionally projects to $\mathrm{SNr}$, whose neurons are also GABAergic (Veenman and Reiner, 1994), receive $\mathrm{SP}^{+}$striatal input (Anderson et al., 1991) and possess AMPA receptor subunits, notably GluR4. In these respects, the SNr neurons in birds resemble SNr and GPi neurons in mammals (Anderson and Reiner, 1990, 1991; Medina et al., 1999; Petralia and Wenthold, 1992; Bernard et al., 1996; Paquet and Smith, 1996). Because both GPi and SNr of mammals project to VA/VL, both the $\mathrm{SP}^{+}$striato-GPi and the $\mathrm{SP}^{+}$striato-SNr pathways are considered parts of the direct motor output system of the basal ganglia (Albin et al., 1989b; Alexander and Crutcher, 1990; DeLong, 1990). Because of its projection to GPi and SNr, STN is the part of the indirect motor output channel of the mammalian basal ganglia by which $\mathrm{ENK}^{+}$striatal neurons influence GPi and $\mathrm{SNr}$ (Albin et al., 1989b; Alexander and Crutcher, 1990; DeLong, 1990). The ALa of birds appears to establish a similar link between the indirect and direct motor output channels of the avian basal ganglia.

As reported previously based on anterograde autoradiographic and retrograde horseradish peroxidase pathway tracing methods (Reiner et al., 1982a), the avian ALa also projects to a cell group within the pretectum, the SpL. Although a mammalian homolog of SpL has not been definitively identified (Reiner et al., 1984, 1998a; Caballero-Bleda et al., 1992; Lagares et al., 1994), several lines of evidence indicate that $\mathrm{SpL}$ in birds is the target of an additional direct striatal output and is thereby analogous to mammalian GPi (Reiner et al., 1984, 1998a; Medina et al., 1999). As is true of the GPi-like cell groups in mammals (i.e., GPi itself and SNr), SpL neurons are GABAergic, receive input from $\mathrm{SP}^{+}$striatal neurons as well as from dorsal pallidal neurons that receive $\mathrm{ENK}^{+}$striatal input (i.e., GPe-like pallidal neurons), and are notably enriched in GluR4 (Karten and Dubbeldam, 1973; Reiner et al., 1982a, 1984; Veenman and Reiner, 1994; Medina and Reiner, 1996, 1997; Pa- 

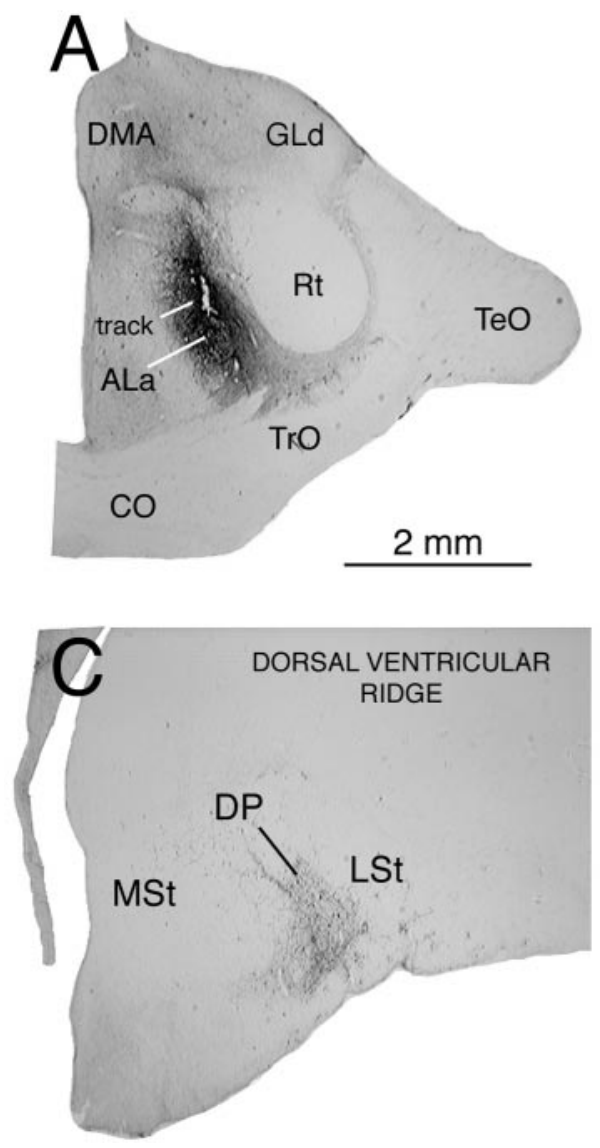
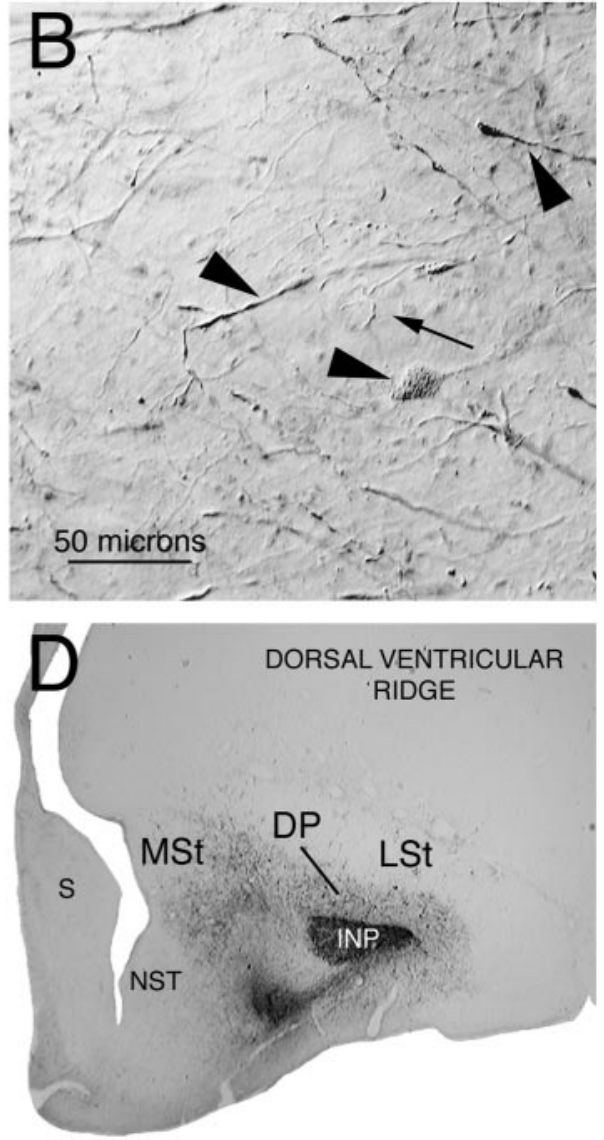

Figure 9. Images showing a BDA10k injection site in ALa in pigeon $(A)$ and the anterograde labeling produced in the dorsal pallidum by this injection, shown at low $(C, D)$ and high $(B)$ magnification. $A$ shows that the injection of BDA was centered on ALa, with the syringe track evident just above ALa. $B$ illustrates that anterogradely labeled fibers and terminals, and some retrogradely labeled perikarya and dendrites, are present in the dorsal pallidum (arrowheads), with fine ALa terminals in the dorsal pallidum contacting cresyl violet-labeled perikarya but not the BDA-labeled neurons. One neuron faintly labeled for cresyl violet that appears to be contacted by $\mathrm{BDA}^{+}$terminals is indicated by the arrow. The intense labeling in the intrapeduncular nucleus (INP) in $D$ reflects perikaryal and dendritic labeling of medium-sized spiny neurons. The intrapeduncular nucleus is striatal in nature (Reiner et al., 1998b), has no single clear mammalian equivalent, and it is uncertain whether intrapeduncular nucleus neurons project to or through the ALa region. The magnification is the same in $A, C$, and $D$. $A L a$, Anterior nucleus of ansa lenticularis; $C O$, optic chiasm; $D M A$, nucleus dorsomedialis anterior thalami; $D P$, dorsal pallidum; $G L d$, dorsal lateral geniculate nucleus; $I N P$, intrapeduncular nucleus; $L S t$, lateral striatum; $M S t$, medial striatum; $N S T$, nucleus of the stria terminalis; $R t$, nucleus rotundus; $S$, septum; $T e O$, optic tectum; $\operatorname{Tr} O$, optic tract.
Figure 10. Images showing low-power $(A, B)$ and high-power $(C, D)$ views of the anterograde labeling produced in the lateral spiriform nucleus $(S p L)$ of the pretectum and the substantia nigra $(S N)$ of the midbrain by the BDA injection into ALa shown in Figure 9. A few retrogradely labeled neurons were observed in the nigra but not in SpL after BDA injections in ALa. The magnification in $A$ is the same as in $B$, and the magnification in $C$ is the same as in $D$. CG, central gray; $H y$, hypothalamus; $P C$, posterior commissure; $S P$, nucleus subpretectalis.
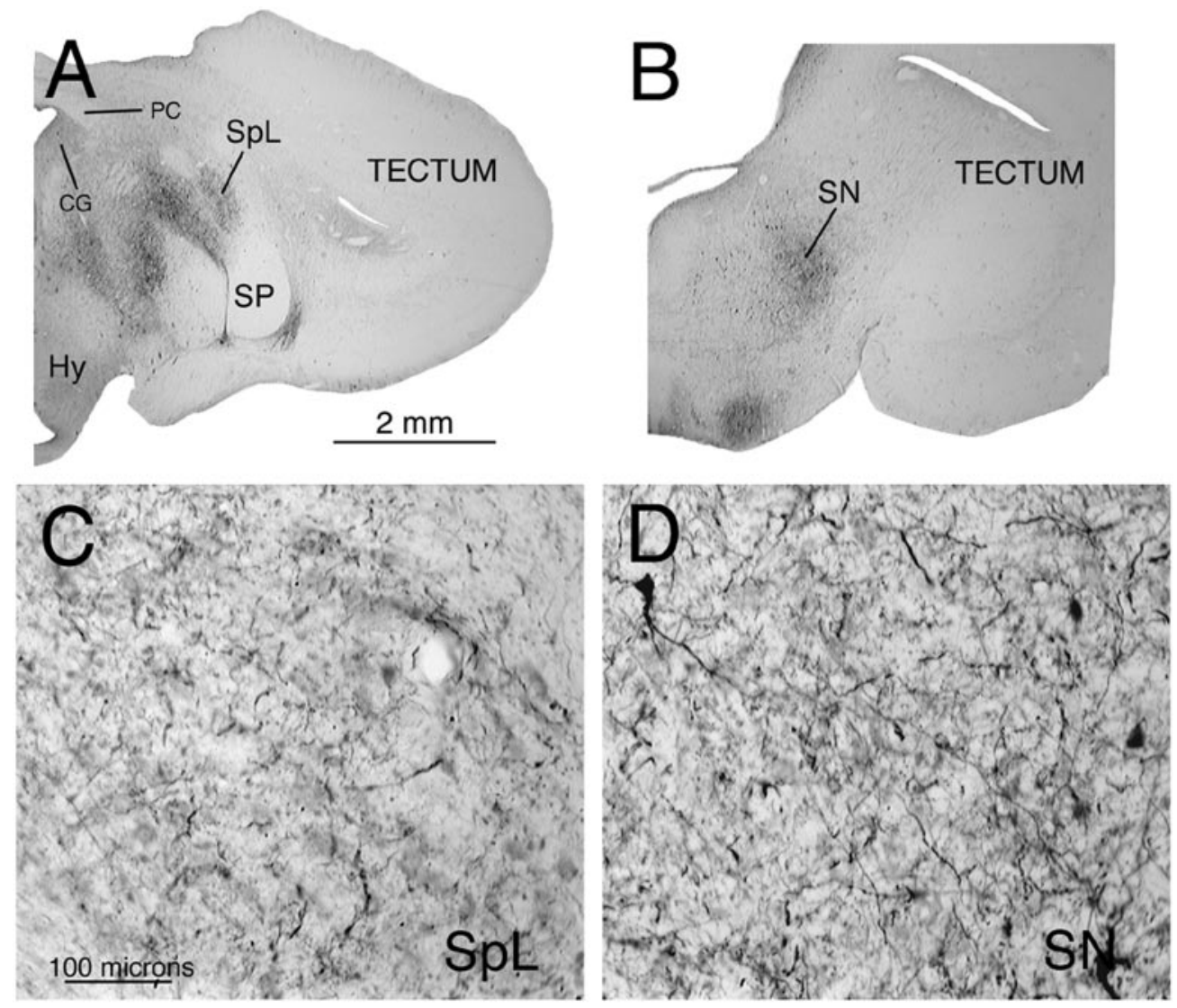

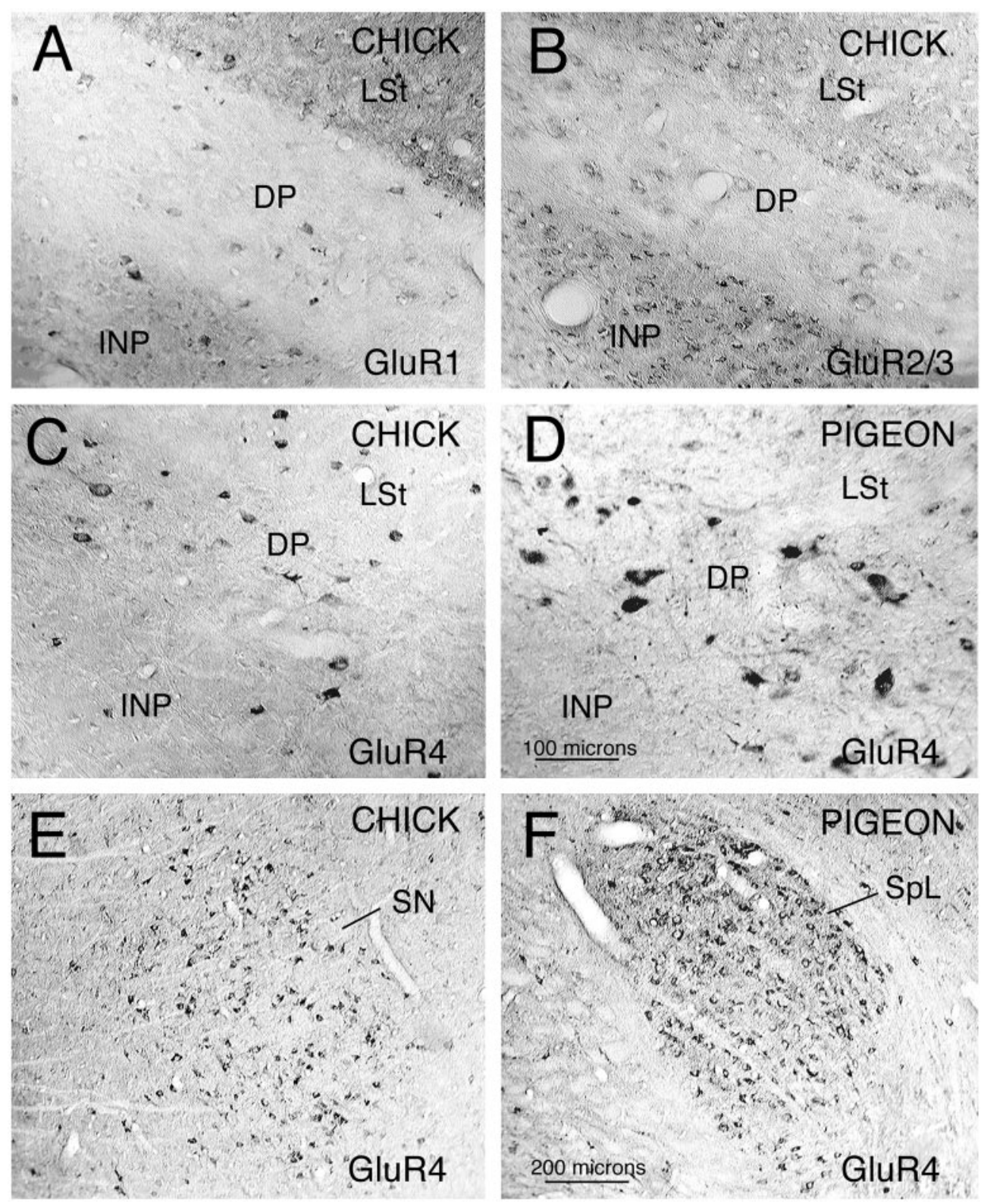
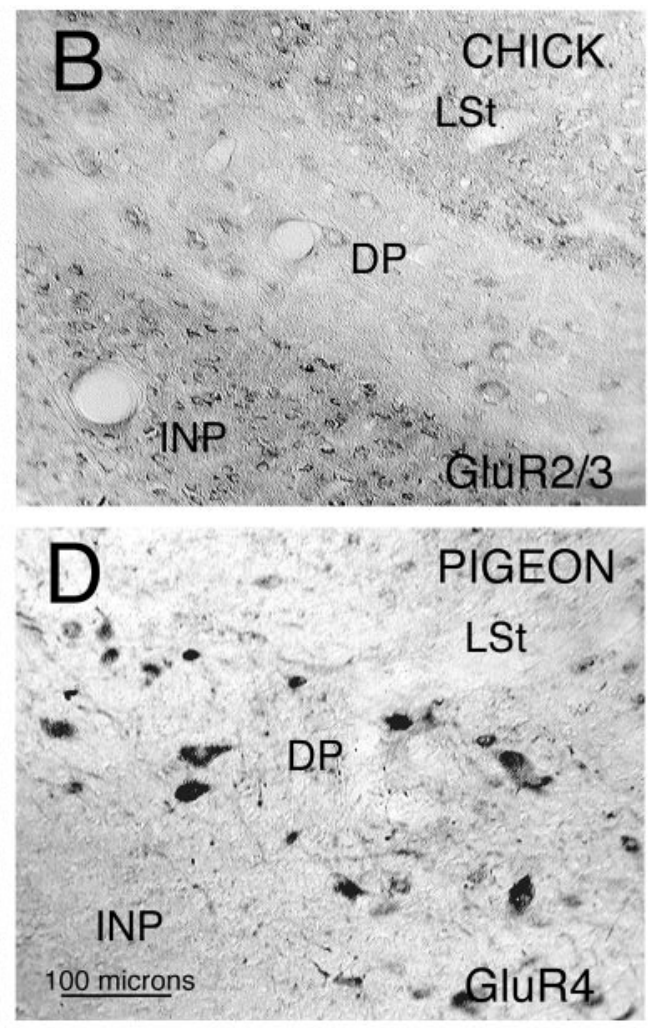

Figure 11. Images showing GluR1labeled perikarya in the dorsal pallidum $(A)$ of chick, GluR2/3-labeled perikarya in the dorsal pallidum of chick $(B)$, GluR4-labeled perikarya in the dorsal pallidum $(C)$ of chick, GluR4-labeled perikarya in dorsal pallidum of pigeon $(D)$, GluR4-labeled perikarya in the pars reticulata of the substantia nigra $(S N)$ of chick $(E)$, and GluR4-labeled perikarya in the lateral spiriform nucleus $(S p L)$ of pigeon $(F)$. The magnification in $A-D$ is the same, and the magnification in $E$ is the same as in $F$. DP Dorsal pallidum; INP, intrapeduncular nucleus; $L S t$, lateral striatum. quet and Smith, 1996; Medina et al., 1999). The SpL itself projects to the tectal layers containing the perikarya and dendrites of neurons with projections to brainstem motor and premotor areas (Reiner et al., 1982a,b, 1984, 1998a; Medina and Reiner, 1995, 1996; Medina et al., 1999). Thus, in both birds and mammals, the motor output circuitry of the striatum consists of the following: (1) output circuits arising from $\mathrm{SP}^{+}$striatal neurons having input to GPi-like pallidal neurons that exert a direct influence on thalamocortical or tectobulbar motor circuits; and (2) output circuits arising from $\mathrm{ENK}^{+}$striatal neurons having input to GPe-like pallidal neurons that exert an indirect effect on thalamocortical or tectobulbar motor circuits via an STN/ALa input to GPi-like neurons of the direct pathways (Fig. 12). Note that living reptiles possess a homolog of the avian basal ganglia-SpL-tectal circuit, which suggests that this circuit was part of basal ganglia circuitry in ancestral amniotes and was lost or de-emphasized in the mammalian lineage, perhaps as a concomitant of the relatively diminished role of the midbrain roof and pretectum in visual functions in the mammalian lineage (Reiner et al., 1984, 1998a).

\section{STN and ALa are involved in suppression of unwanted movements}

Whereas large unilateral STN lesions in primates produce sustained contralateral hemiballism (Carpenter et al., 1950; Albin et al., 1989b), STN lesions in rats produce only transient contralateral hyperkinesia and rotation (Kafetzopoulos and Papadopoulos, 1983; Piallat et al., 1996). As in rats, we found that large unilateral lesions of ALa transiently produce contraversive hyperkinesia and spontaneous rotation in pigeons. Additionally, our results indicate that apomorphine treatment of birds with unilateral ALa lesions causes the birds to rotate to the side of the lesion. Rats with STN lesions also exhibit rotation to the side of the lesion after apomorphine treatment (Kafetzopoulos and Papadopoulos, 1983; Piallat et al., 1996). The contralateral hemiballism in mammals appears to occur because the cortical motor areas receiving input from the ipsilateral basal ganglia control contralateral limbs. The spontaneous movement toward the limb affected by the STN lesion may occur because the ballism on that side impairs motor output on that side, leaving it less able to counterbalance the normal movement output of the opposite limb controlled by the intact basal ganglia. The basis of the ipsiversive apomorphine-induced rotation after an STN lesion is less clear, particularly because apomorphine induces rotation away from the lesioned side in a rat with unilateral destruction of nigral dopaminergic neurons (Hifti et al., 1980; Zeng et al., 1995). In any case, the similar behavioral effects of STN lesions in rats and ALa lesions in pigeons suggest a similar functional role of STN and ALa in suppression of unwanted 


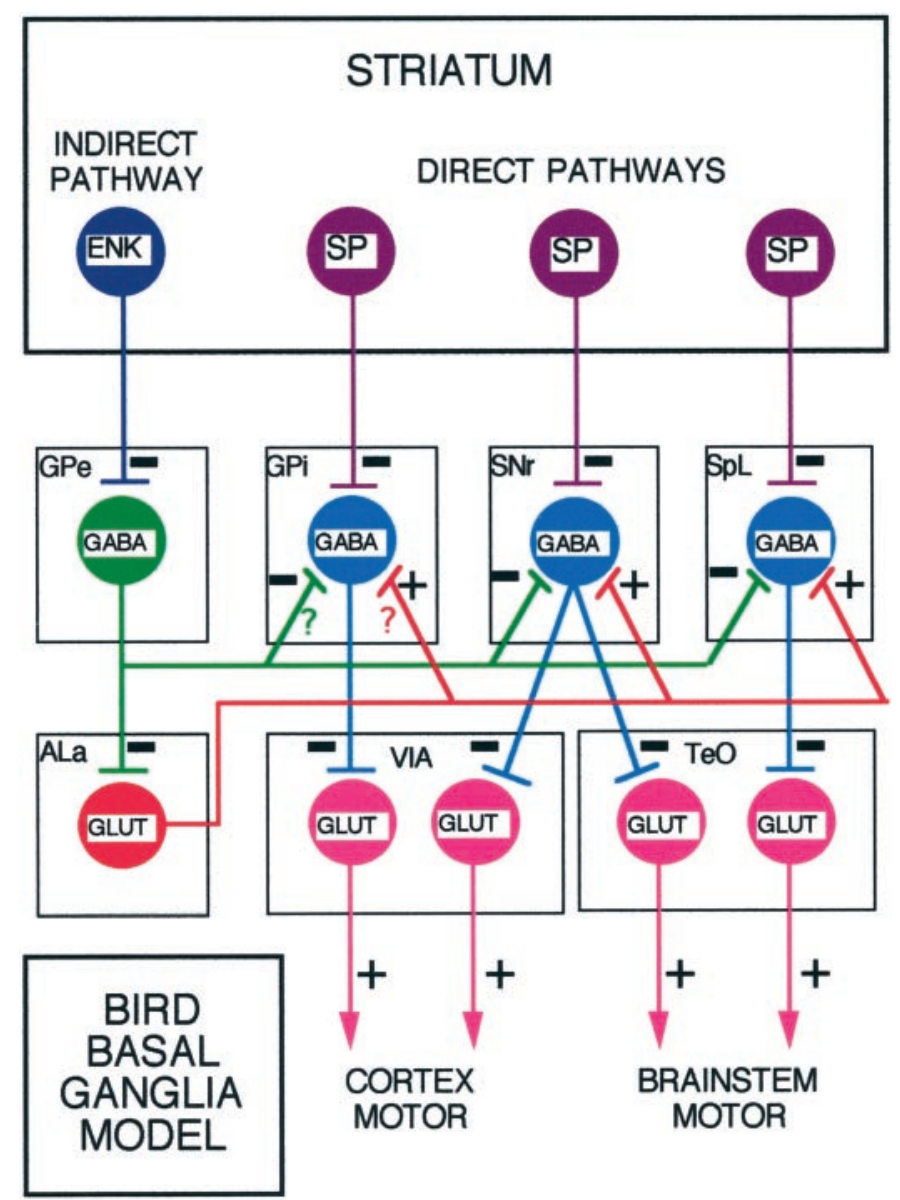

Figure 12. Circuit diagram of the functional organization of the basal ganglia in birds. The + and - indicate whether specific projections use an excitatory $(+)$ or inhibitory $(-)$ neurotransmitter. The characteristic transmitter used by each major type of projection neuron is also shown. As in mammals, the striatal and pallidal output circuitry of birds appears organized into direct $\mathrm{SP}^{+}$striatal outputs to pallidal neurons promoting movement and $\mathrm{ENK}^{+}$striatal outputs to pallidal neurons inhibiting unwanted movement. The pallidal neurons of the indirect pathway have direct outputs to the targets of the $\mathrm{SP}^{+}$striatal neurons (i.e., GPi, $\mathrm{SNr}$, and $\mathrm{SpL}$ ) and indirect outputs to these same targets via ALa (i.e., the subthalamic nucleus of birds). Because neurons of the $\mathrm{ENK}^{+}$pathway indirectly project to the same targets as neurons of $\mathrm{SP}^{+}$pathways, the ENK pathway has been called the indirect pathway in mammals. In mammals, $\mathrm{SP}^{+}$neurons target two populations of pallidal-type neurons ( $\mathrm{GPi}$ and $\mathrm{SNr}$ ), whereas in birds three are targeted $(\mathrm{GPi}, \mathrm{SNr}$, and $\mathrm{SpL})$. It is not yet certain, however, for birds whether ALa projections and GPe-type pallidal projection neurons (i.e., receiving ENK input) specifically project to GPi type neurons (i.e., receiving SP input) within the pallidum. It is also uncertain whether GPe-like neurons in the avian dorsal pallidum have a projection to GPi-type neurons of the dorsal pallidum. Such a projection has been demonstrated in mammals (Hazrati et al., 1990; Kincaid et al., 1990; Smith et al., 1994; Shink et al., 1996). $A L a$, Anterior nucleus of the ansa lenticularis; ENK, enkephalin; $G L U T$, glutamate; $G P e$, external segment of globus pallidus; $G P i$, internal segment of globus pallidus; $S N r$, substantia nigra pars reticulata; $S P$, substance P; $S p L$, nucleus spiriformis lateralis; $T e O$, optic tectum; VIA, ventrointermediate thalamic area.

movements and a similar interplay between the direct and indirect pathways in mammals and birds in effecting basal ganglia-mediated influences on movement (Reiner et al., 1998a; Medina et al., 1999) (Fig. 12).

\section{Evolutionary implications: reptiles}

The dorsal pallidum in reptiles projects to a nucleus in the subthalamus called the anterior entopeduncular nucleus (ENa) (Brauth and Kitt, 1980; Brauth, 1988). The ENa neurons are glutamatergic (Fowler et al., 1999) and project to dorsal pallidum and $\mathrm{SNr}$ (Brauth et al., 1978; Brauth and Kitt, 1980), thus resembling the mammalian STN and avian ALa (Albin et al., 1989a,b; DeLong,
1990; Kitai and Kita, 1987; Smith and Parent, 1988; Smith et al., 1994; Shink et al., 1996). Additionally, dorsal pallidal neurons in reptiles possess AMPA-type glutamate receptors, as in birds and mammals, consistent with a glutamatergic input from ENa to dorsal pallidum (Bernard et al., 1996; Götz et al., 1997; Fowler et al., 1999). Finally, the topographic location of ENa at the border of the hypothalamus and its apparent segmental location in prosomere 4 closely resembles that of avian ALa and mammalian STN (Puelles and Medina, 1994). This evidence of a region comparable with ALa and STN in the forebrain of modern reptiles reinforces our proposed homology of ALa and STN and suggests that both evolved from a comparable region in prosomere 4 in stem amniotes. This proposed homology also implies that the basal ganglia was organized into direct and indirect motor output pathways in the stem amniotes that were the common ancestors of living reptiles, birds, and mammals (Reiner et al., 1998a). Although detailed delineation of the putative direct and indirect pathways of the basal ganglia has not been performed for living reptiles, the existing anatomical, neurochemical, pharmacological, and behavioral data for reptiles are consistent with the premise they are present (Reiner et al., 1980; Reiner and Carraway, 1987; Anderson and Reiner, 1990; Reiner and Anderson, 1990).

\section{Evolutionary implications: anamniotes}

In amphibians, the basal ganglia has a projection to a subthalamic region that projects back to the basal ganglia (Wilczynski and Northcutt, 1983b; Marin et al., 1997a,b). It is unknown whether the neurons of this subthalamic region are glutamatergic, project to a pallidum and $\mathrm{SNr}$, and are located in the basal plate of prosomere 4 (Puelles et al., 1996). The available data suggest that the basal telencephalon in bony and cartilaginous fish may also project to the subthalamus, but the precise source of the projection and the precise projection target of this subthalamic region are uncertain (Airhart et al., 1988; Smeets, 1990). Although a subthalamic nucleus has, thus, not been identified in anamniotes, both $\mathrm{SP}^{+}$and $\mathrm{ENK}^{+}$striatal neurons are present in the basal ganglia of all jawed anamniote species studied (Reiner and Northcutt, 1987, 1992; Northcutt et al., 1988; Reiner et al., 1998a). On this basis, it might be expected that the basal ganglia possess direct and indirect motor output circuits in all jawed anamniotes.

\section{REFERENCES}

Airhart MJ, Shirk JO, Kriebel RM (1988) Telencephalic projections to the goldfish hypothalamus: an anterograde degeneration study. Brain Res Bull 20:503-514.

Albin RL, Aldridge JW, Young AB, Gilman S (1989a) Feline subthalamic nucleus neurons contain glutamate-like but not GABA-like or glycinelike immunoreactivity. Brain Res 491:185-188.

Albin RL, Young AB, Penney JB (1989b) The functional anatomy of basal ganglia disorders. Trends Neurosci 12:366-375.

Albin RL, Reiner A, Anderson KD, Penney JB, Young AB (1990a) Striatal and nigral neuron subpopulations in rigid Huntington's disease: implications for the functional anatomy of chorea and rigidity-akinesia. Ann Neurol 27:357-365.

Albin RL, Young AB, Penney JB, Handelin B, Balfour R, Anderson KD, Markel DS, Tourtellotte WW, Reiner A (1990b) Striatal enkephalinergic abnormalities and $N$-methyl-D-aspartate receptor abnormalities in a case of presymptomatic Huntington's disease. N Engl J Med 322:1293-1298.

Albin RL, Reiner A, Anderson KD, Dure IV LS, Handelin B, Balfour R, Whetsell Jr WO, Penney JB, Young AB (1992) Preferential loss of striato-external pallidal and striato-nigral projection neurons in presymptomatic Huntington's disease. Ann Neurol 31:425-430.

Alexander GE, Crutcher MD (1990) Functional architecture of basal ganglia circuits: neural substrates of parallel processing. Trends Neurosci $13: 266-271$

Altman J, Bayer SA (1978a) Development of the diencephalon in the rat. I. Autoradiographic study of the time of origin and settling patterns of neurons of the hypothalamus. J Comp Neurol 182:945-972.

Altman J, Bayer SA (1978b) development of the diencephalon in the rat. II. Correlation of the embryonic development of the hypothalamus with the time of origin of its neurons. J Comp Neurol 182:973-994.

Anderson KD, Reiner A (1990) Extensive co-occurrence of substance P and dynorphin in striatal projection neurons: an evolutionarily conserved feature of basal ganglia organization. J Comp Neurol 295:339-369.

Anderson KD, Reiner A (1991) Striatonigral projection neurons: a retro- 
grade labeling study of the percentages that contain substance $\mathrm{P}$ or enkephalin. J Comp Neurol 303:658-673.

Anderson KD, Karle EJ, Reiner A (1991) Ultrastructural single- and double-label immunohistochemical studies of substance P-containing terminals and dopaminergic neurons in the substantia nigra in pigeons. J Comp Neurol 309:341-362.

Balk JH, Picetti R, Salardi A, Thirlet G, Dierich A, Depaulis A, Le Meur M, Borrelli E (1995) Parkinsonian-like locomotor impairment in mice lacking dopamine D2 receptors. Nature 377:424-428.

Bernard V, Gardiol A, Fachueux B, Bloch B, Agid Y, Hirsch EC (1996) Expression of glutamate receptors in the human and rat basal ganglia: effect of the dopaminergic denervation on AMPA receptor gene expression in the striatopallidal complex in Parkinson's disease and rat with 6OHDA lesion. J Comp Neurol 368:553-568.

Brauth SE (1988) The organization and projections of the paleostriatal complex in Caiman crocodilus. In: The forebrain of reptiles. Current concepts of structure and function (Schwerdtfeger WK, Smeets WJAJ, eds), pp 60-76. Basel: Karger.

Brauth SE, Kitt CA (1980) The paleostriatal system of Caiman crocodilus. J Comp Neurol 189:437-465.

Brauth SE, Ferguson JL, Kitt CA (1978) Prosencephalic pathways related to the paleostriatum of the pigeon (Columba livia). Brain Res 147:205-221.

Bulfone A, Puelles L, Porteus MH, Frohman MA, Martin GR, Rubenstein JLR (1993) Spatially restricted expression of Dlx-1, Dlx-2 (Tes-1), Gbx-2, and Wnt-3 in the embryonic day 12.5 mouse forebrain defines potential transverse and longitudinal segmental boundaries. J Neurosci 13:3155-3172.

Caballero-Bleda M, Fernandez B, Puelles L (1992) The pretectal complex of the rabbit: distribution of acetylcholinesterase and reduced nicotinamide adenine dinucleitide diaphorase activities. Acta Anat 144:7-16.

Carpenter MB, Whittier JR, Mettler FA (1950) Analysis of choreoid hyperkinesia in rhesus monkey. Surgical and pharmacological analysis of hyperkinesia resulting after lesions in the subthalamic nucleus of Luys. J Comp Neurol 92:293-331.

Chen Q, Veenman CL, Reiner A (1996) Cellular expression of ionotropic glutamate receptor subunits on specific striatal neuron types and its implication for striatal vulnerability in glutamate receptor-mediated excitotoxicity. Neuroscience 73:715-731.

Chen Q, Veenman CL, Knopp K, Yan Z, Medina L, Song WJ, Surmeier DJ, Reiner A (1998) Evidence for the preferential localization of GluR1 subunits of AMPA receptors to the dendritic spines of medium spiny neurons in rat striatum. Neuroscience 83:749-761.

Conti F, Rustioni A, Petrusz P, Towle AC (1987) Glutamate-positive neurons in the somatic sensory cortex of rats and monkeys. J Neurosci 7:1887-1901.

DeLong MR (1990) Primate models of movement disorders of basal ganglia origin. Trends Neurosci 13:281-285.

Fowler M, Medina L, Reiner A (1999) Immunohistochemical localization of NMDA- and AMPA-type glutamate receptor subunits in the basal ganglia of red-eared turtles. Brain Behav Evol 54:276-289.

Fritzsch B (1993) Fast axonal diffusion of 3000 molecular weight dextran amine. J Neurosci Methods 50:95-103.

Gerfen CR (1992) The neostriatal mosaic: multiple levels of compartmental organization in the basal ganglia. Ann Rev Neurosci 15:285-320.

Götz T, Kraushaar U, Geiger J, Lübke J, Berger T, Jones P (1997) Functional properties of AMPA and NMDA receptors expressed in identified types of basal ganglia neurons. J Neurosci 17:204-215.

Graybiel AM (1990) Neurotransmitters and neuromodulators in the basal ganglia. Trends Neurosci 13:244-253.

Hamburger V, Hamilton HL (1951) A series of normal stages in the development of the chick embryo. J Morphol 88:49-92.

Hazrati LN, Parent A (1992) Differential patterns of arborization of striatal and subthalamic fibers in the two pallidal segments in primates. Brain Res 598:311-315.

Hazrati LN, Parent A, Mitchell S, Haber SN (1990) Evidence for interconnections between the two segments of the globus pallidus in primates: a PHA-L anterograde tracing study. Brain Res 533:171-175.

Herrera-Marschitz M, Ungerstedt U (1984) Evidence that striatal efferents relate to different dopamine receptors. Brain Res 323:269-278.

Hifti F, Melamed E, Wurtman RJ (1980) Partial lesions of the dopaminergic nigrostriatal system in rat brain: biochemical characterization. Brain Res 195:123-137.

Hodos W, Karten HJ (1966) Brightness and pattern discrimination deficits in the pigeon after lesions in the nucleus rotundus. Exp Brain Res 2:151-167.

Hutchison WD, Levy R, Dostrovsky JO, Lozano AM, Lang AE (1997) Effects of apomorphine on globus pallidus neurons in Parkinsonian patients. Ann Neurol 42:767-775.

Kafetzopoulos E, Papadopoulos G (1983) Turning behavior after unilateral lesion of the subthalamic nucleus in the rat. Behav Brain Res 8:217-223.

Kaneko T, Saeki K, Lee T, Mizuno N (1996) Improved retrograde axonal transport and subsequent visualization of tetramethylrhodamine (TMR)dextran amine by means of an acidic injection vehicle and antibodies against TMR. J Neurosci Methods 65:157-165.

Karten HJ, Dubbeldam JL (1973) The organization and projections of the paleostriatal complex in the pigeon (Columba livia). J Comp Neurol 148:61-90.

Karten HJ, Hodos W (1967) A stereotaxic atlas of the brain of the pigeon (Columba livia). Baltimore: Johns Hopkins UP.

Karten HJ, Revzin AM (1966) The afferent connections of the nucleus rotundus in the pigeon. Brain Res 2:368-377.

Karten HJ, Revzin AM (1970) Telencephalic projections of the nucleus rotundus in the pigeon (Columba livia). J Comp Neurol 140:35-52.

Keyser A (1972) The development of the diencephalon of the Chinese hamster. An investigation of the validity of the criteria of subdivision of the brain. Acta Anat [Suppl 59] 83:1-181.

Kincaid AE, Newman SW, Young AB, Penney JB (1990) Evidence for a projection from the globus pallidus to the entopeduncular nucleus in the rat. Neurosci Lett 128:121-125.

Kita H, Kitai ST (1987) Efferent projections of the subthalamic nucleus in the rat: light and electron microscopic analysis with the PHA-L method. J Comp Neurol 260:435-452.

Kitai ST, Kita H (1987) Anatomy and physiology of the subthalamic nucleus: a driving force of the basal ganglia. In: The basal ganglia II. Structure and function: current concepts (Carpenter MB, Jaraman A, eds), pp 357-373. New York: Plenum.

Lagares C, Caballero-Bleda M, Fernandez B, Puelles L (1994) reciprocal connections between the rabbit suprageniculate pretectal nucleus and the superior colliculus: tracer study with horseradish peroxidase and fluorogold. Visual Neurosci 11:347-353.

Lumsden A, Keynes R (1989) Segmental patterns of neuronal development in the chick hindbrain. Nature 337:424-428.

Marin F, Puelles L (1994) Morphological fate of rhombomeres in quail/ chick chimeras: a segmental analysis of hindbrain nuclei. Eur J Neurosci 7:1714-1738.

Marin O, Gonzalez A, Smeets WJAJ (1997a) Basal ganglia organization in amphibians: afferent connections to the striatum and the nucleus accumbens. J Comp Neurol 378:16-49.

Marin O, Gonzalez A, Smeets WJAJ (1997b) Basal ganglia organization in amphibians: efferent connections of the striatum and the nucleus accumbens. J Comp Neurol 380:23-50.

Medina L, Reiner A (1995) Neurotransmitter organization and connectivity of the basal ganglia in vertebrates: implications for the evolution of the basal ganglia. Brain Behav Evol 46:235-258.

Medina L, Reiner A (1996) Immunohistochemical characterization of the striatal input to neurons of the dorsal pallidum in pigeon, with particular emphasis on pallido-thalamic neurons. Soc Neurosci Abstr 22:674.

Medina L, Reiner A (1997) The efferent projections of the dorsal and ventral pallidal parts of the pigeon basal ganglia, studied with biotinylated dextran amine. Neuroscience 81:773-802.

Medina L, Reiner A (2000) Do birds possess homologues of mammalian primary visual, somatosensory and motor cortices? Trends Neurosci 23:1-12.

Medina L, Figueredo-Cardenas G, Reiner A (1996) Differential abundance of superoxide dismutase in interneurons versus projection neurons in patch versus matrix neurons in monkey striatum. Brain Res 708:59-70.

Medina L, Veenman CL, Reiner A (1997) New evidence for an avian dorsal thalamic center comparable to the mammalian VA/VL nuclei. J Comp Neurol 384:86-108.

Medina L, Jiao Y, Reiner A (1999) The functional anatomy of the basal ganglia in birds. Eur J Morphol 37:160-165.

Northcutt RG, Reiner A, Karten HJ (1988) An immunohistochemical study of the telencephalon of the spiny dogfish, Squalus acanthias. J Comp Neurol 277:250-267.

Ottiger HP, Gerfin-Moser A, Del Principe F, Dutly F, Streit P (1995) Molecular cloning and differential expression patterns of avian glutamate receptor mRNAs. J Neurochem 64:2413-2426.

Paperna T, Lamed Y, Teichberg VI (1996) cDNA cloning of chick brain $\alpha$-amino-3-hydroxy-4-isoxazolepropionic acid receptors reveals conservation of structure, function and post-transcriptional processes with mammalian receptors. Mol Brain Res 36:101-113.

Paquet M, Smith Y (1996) Differential localization of AMPA glutamate receptor subunits in the two segments of the globus pallidus and the substantia nigra reticulata in the squirrel monkey. Eur $\mathbf{J}$ Neurosci 8:229-233.

Petralia RS, Wenthold RJ (1992) Light and electron immunocytochemical localization of AMPA-selective glutamate receptors in the rat brain. J Comp Neurol 318:329-354.

Piallat B, Benazzouz A, Benabid AL (1996) Subthalamic nucleus lesion in rats prevents dopaminergic nigral neuron degeneration after striatal 6-OHDA injection: behavioral and immunohistochemical studies. Eur J Neurosci 8:1408-1414.

Pombal MA, Puelles L (1999) Prosomeric map of the lamprey forebrain based on calretinin immunocytochemistry, nissl stain and ancillary markers. J Comp Neurol 414:391-422.

Puelles L (1995) A segmental morphological paradigm for understanding vertebrate forebrains. Brain Behav Evol 46:319-337.

Puelles L, Medina L (1994) Development of neurons expressing tyrosine hydroxylase and dopamine in the chicken brain: a comparative segmental analysis. In: Phylogeny and development of catecholamine systems in the CNS of vertebrates (Smeets WJAJ, Reiner A, eds), pp 381-404. Cambridge, UK: Cambridge UP. 
Puelles L, Rubenstein JLR (1993) Expression patterns of homeobox and other putative regulatory genes in the embryonic mouse forebrain suggest regulatory genes in the embryonic mouse forebrain suggest a neuromeric organization. Trends Neurosci 16:472-479.

Puelles L, Amat JA, Martínez-de-la-Torre M (1987) Segment-related, mosaic neurogenetic pattern in the forebrain and mesencephalon of early chick embryos. I. Topography of AChE-positive neuroblasts up to stage HH18. J Comp Neurol 266:247-268.

Puelles L, Milan FJ, Martinez-de-la-Torre M (1996) A segmental map of architectonic subdivisions in the diencephalon of the frog Rana perezi: acetylcholinesterase-histochemical observations. Brain Behav Evol 47:279-310.

Puelles L, Kuwana E, Puelles E, Rubenstein JLR (1999) Comparison of the mammalian and avian telencephalon from the perspective of gene expression data. Eur J Morphol 37:139-150.

Puelles L, Kuwana E, Puelles E, Bulfone A, Shimamura K, Keleher J, Smiga S, Rubenstein JLR (2000) Pallial and subpallial derivatives in the embryonic chick and mouse telencephalon, traced by the expression of the genes Dlx-2, Emx-1, Nkx-2.1, Pax-6 and Tbr-1. J Comp Neurol 424:409-438.

Ravindranathan A, Parks TN, Rao MS (1996) Flip and flop isoforms of chick brain AMPA subunits: cloning and analysis of expression patterns. NeuroReport 7:2707-2711.

Redies C, Ast M, Nakagawa S, Takeichi M, Martinez-de-la-Torre M, Puelles L (2000) Morphological fate of diencephalic prosomeres and their subdivisions revealed by mapping cadherin expression. J Comp Neurol 421:481-514.

Reiner A (1994) Catecholaminergic innervation of the basal ganglia in mammals: anatomy and function. In: Phylogeny and development of catecholamine systems in the CNS of vertebrates (Smeets WJAJ, Reiner A, eds), pp 247-272. Cambridge, UK: Cambridge UP.

Reiner A, Anderson KD (1990) The patterns of neurotransmitter and neuropeptide co-occurrence among striatal projection neurons: conclusions based on recent findings. Brain Res Rev 15:251-265.

Reiner A, Anderson KD (1993) Co-ocurrence of $\gamma$-aminobutyric acid, parvalbumin and the neurotensin-related neuropeptide LANT6 in pallidal, nigral and striatal neurons in pigeons and monkeys. Brain Res 624:317-325.

Reiner A, Carraway RE (1987) Immunohistochemical and biochemical studies on Lys8-Asn9-neurotensin8-13(LANT6)-related peptides in the basal ganglia of pigeons, turtles, and hamsters. J Comp Neurol 257:453-476.

Reiner A, Karten HJ (1982) Laminar distribution of the cells of origin of the descending tectofugal pathways in the pigeon (Columba livia). J Comp Neurol 204:165-187.

Reiner A, Northcutt RG (1987) An immunohistochemical study of the telencephalon of the African lungfish. J Comp Neurol 256:463-481.

Reiner A, Northcutt RG (1992) An immunohistochemical study of the telencephalon of the Senegal Bichir (Polypterus senegalus). J Comp Neurol 319:359-386.

Reiner A, Brauth SE, Kitt CA, Karten HJ (1980) Basal ganglionic pathways to the tectum: studies in reptiles. J Comp Neurol 193:565-589.

Reiner A, Brecha NC, Karten HJ (1982a) Basal ganglia pathways to the tectum: the afferent and efferent connections of the lateral spiriform nucleus of pigeon. J Comp Neurol 208:16-36.

Reiner A, Karten HJ, Brecha NC (1982b) Enkephalin-mediated basal ganglia influences over the optic tectum: immunohistochemistry of the tectum and the lateral spiriform nucleus in pigeon. J Comp Neurol 208:37-53.

Reiner A, Brauth SE, Karten HJ (1984) Evolution of the amniote basal ganglia. Trends Neurosci 7:320-325.

Reiner A, Albin RL, Anderson KD, D'Amato CJ, Penney JB, Young AB (1988) Differential loss of substance P-containing striatopallidal and enkephalin-containing striatopallidal projections in Huntington's disease. Proc Natl Acad Sci USA 85:5733-5737.
Reiner A, Veenman CL, Honig MG (1993) Anterograde tracing using biotinylated dextran amine. Neurosci Prot 93-050-14-01-14.

Reiner A, Medina L, Veenman CL (1998a) Structural and functional evolution of the basal ganglia in vertebrates. Brain Res Rev 28:235-284.

Reiner A, Perera M, Paullus R, Medina L (1998b) Immunohistochemical localization of DARPP-32 in striatal projection neurons and striatal interneurons in pigeons. J Chem Neuroanat 16:17-33.

Reiner A, Medina L, Haber SN (1999) The distribution of dynorphinergic terminals in striatal target regions in comparison to the distribution of substance P-containing and enkephalinergic terminals in monkeys and humans. Neuroscience 88:775-793.

Rubenstein JLR, Martinez S, Shimamura K, Puelles L (1994) The embryonic vertebrate forebrain: the prosomeric model. Science 266:578-580.

Rubenstein JLR, Shimamura K, Martinez S, Puelles L (1998) Regionalization of the prosencephalic neural plate. Ann Rev Neurosci 21:445-477.

Shimamura K, Hartigan DJ, Martinez S, Puelles L, Rubenstein JLR (1995) Longitudinal organization of the anterior neural plate and neural tube. Development 121:3923-3933.

Shimamura K, Martinez S, Puelles L, Rubenstein JLR (1997) Patterns of gene expression in the neural plate and neural tube subdivide the embryonic forebrain into transverse and longitudinal domains. Dev Neurosci 19:88-96.

Shink E, Bevan MD, Bolam JP, Smith Y (1996) The subthalamic nucleus and the external pallidum: two tightly interconnected structures that control the output of the basal ganglia in the monkey. Neuroscience 73:335-357.

Smeets WJAJ (1990) The telencephalon of cartilaginous fishes. In: Cereb cortex, Vol 8A, Pt 1, Nonmammalian vertebrates (Jones EG, Peters A, eds), pp 3-30. New York: Plenum.

Smith Y, Parent A (1988) Neurons of the subthalamic nucleus in primates display glutamate but not GABA immunoreactivity. Brain Res 453:353-356.

Smith Y, Wichmann T, DeLong MR (1994) Synaptic innervation of neurones in the internal pallidal segment by the subthalamic nucleus and the external pallidum in monkeys. J Comp Neurol 343:297-318.

Vaage S (1969) The segmentation of the primitive neural tube in chick embryos (Gallus domesticus). A morphological, histochemical and autoradiographical investigation. Adv Anat Embryol Cell Biol 41:1-88.

Veenman CL, Reiner A (1994) The distribution of GABA-containing perikarya, fibers, and terminals in the forebrain and midbrain of pigeons, with particular reference to the basal ganglia and its projection targets. J Comp Neurol 339:209-250.

Veenman CL, Reiner A, Honig MG (1992) Biotinylated dextran amine as an anterograde tracer for single- and double-label studies. J Neurosci Methods 41:239-254.

Veenman CL, Karle EJ, Anderson KD, Reiner A (1995) Thalamostriatal projections neurons in birds utilize LANT6 and neurotensin: a light and electron microscopic double-labeling study. J Chem Neuroanat 9:1-16.

Veenman CL, Medina L, Reiner A (1997) Avian homologues of the mammalian intralaminar, mediodorsal and midline thalamic nuclei: immunohistochemical and hodological evidence. Brain Behav Evol 49:78-98.

Wilczynski W, Northcutt RG (1983b) Connections of the bullfrog striatum: efferent projections. J Comp Neurol 214:333-343.

Xu M, Moratalla R, Gold LH, Hiroi N, Koob GF, Graybiel AM, Tonegawa S (1994) Dopamine D1 receptor mutant mice are deficient in striatal expression of dynorphin and in dopamine-mediated behavioral responses. Cell 79:729-742.

Zeng BY, Jolkkonen J, Jenner P, Marsden CD (1995) Chronic L-DOPA treatment differentially regulates gene expression of glutamate decarboxylase, preproenkephalin and preprotachykinin in the striatum of 6-hydroxydopamine-lesioned rat. Neuroscience 66:19-28. 